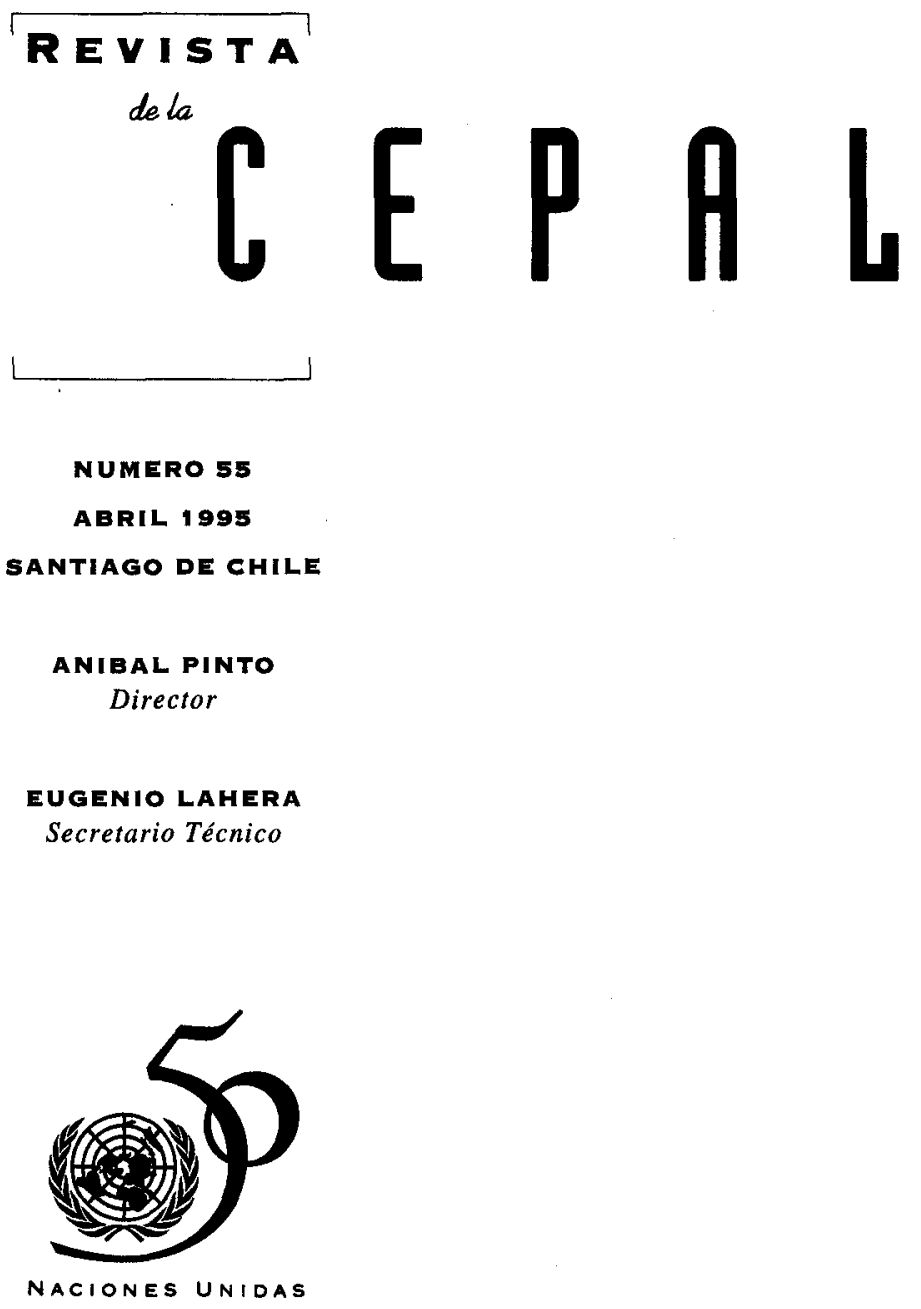


Una síntesis de la propuesta de la CEPAL

Eugenio Lahera, Ernesto Ottone y Osvaldo Rosales

Consolidación de la paz después de los conflictos:

un desafío para las Naciones Unidas

Graciana del Castillo

Descentralización y democracia: el nuevo municipio latinoamericano

Eduardo Palma

Economía política del proteccionismo después de la Ronda Uruguay

José Tavares

Política comercial e inserción internacional.

Una perspectiva latinoamericana

Marta Bekerman y Pablo Sirlin

Movimientos de capital y financiamiento externo

Benjamín Hopenhayn

Impacto de la política cambiaria y comercial sobre el desempeño

exportador en los años ochenta

Graciela Moguillansky

Situación y perspectivas ambientales en América Latina y el Caribe

Nicolo Gligo

Expectativas de la juventud y el desarrollo rural

Martine Dirven

Empresas transnacionales y cambios estructurales en la industria de Argentina, Brasil, Chile y México

Ricardo Bielschowsky y Giovanni Stumpo

El Salvador: política industrial, comportamiento empresarial y perspectivas

Roberto Salazar

El cambio tecnológico en los análisis estructuralistas

Armando Kuri

Orientaciones para los colaboradores de la Revista de la CEPAL 


\section{Empresas transnacionales y cambios estructurales en la industria de Argentina, Brasil, Chile y México}

\section{Ricardo A. Bielschowsky Giovanni Stumpo}

Oficial de Asuntos sobre

Empresas Transnacionales,

Unidad Conjunta CEPAL

UNCTAD de Empresas

Transnacionales, CEPAL.

Experto Asociado, División de Desarrollo Empresarial, CEPAL.
El tema central de este artículo es el papel que desempeñaron las empresas transnacionales en la reestructuración industrial de la Argentina, Brasil, Chile y México, desde fines del período de la sustitución de importaciones hasta comienzos de los años noventa. A partir de estudios publicados recientemente sobre la profunda transformación que ha experimentado la industria manufacturera latinoamericana tras los efectos de la crisis económica y del proceso de apertura, se utiliza aquí un programa computacional elaborado por la División de Desarrollo Productivo y Empresarial de la CEPAL, para examinar los cambios que se han producido en la estructura productiva (composición sectorial y eficiencia) y en la inserción internacional del sector. A fin de evidenciar el papel relevante que tuvieron las empresas transnacionales en esos procesos, la producción manufacturera se ha clasificado y analizado según si la participación en ella de dichas empresas fue "protagónica", "coadyuvante" o "marginal". Merced a esta clasificación de los sectores industriales, se pudo probar que las reacciones de las empresas transnacionales y los procesos de reestructuración del aparato industrial presentaron modalidades muy diferentes en cada país considerado. Esas modalidades o "estilos" derivan de la combinación de tres grupos de factores que son propios de cada país: estructurales, macroeconómicos e institucionales. 


\section{I}

\section{Introducción}

Este artículo versa sobre el papel que tuvieron las empresas transnacionales (ETs) en la transformación estructural de la industria manufacturera, en Argentina a partir de 1978, en Brasil desde 1981, en Chile desde 1973, y en México desde 1982. Las distintas fechas corresponden al primer año que siguió al estancamiento de la industria de cada uno de esos países en el "modelo" de sustitución de importaciones. ${ }^{1}$

La casi totalidad de las grandes ETs manufactureras que existen actualmente en la región se instalaron durante el proceso sustitutivo. La presencia masiva de capital extranjero en el curso de ese proceso facilita el análisis combinado de los cambios estructurales que experimentó la industria de la región y la participación en ellos de las ETs. Los casos de ajuste o reconversión a los entornos de crisis y de apertura comercial coinciden con la historia misma de la reestructuración de los sistemas manufactureros en esos países. Es más, definen, incluso, muchos de los rasgos centrales de dicha reestructuración. ${ }^{2}$

En el artículo se combinan cinco proposiciones perfectamente complementarias:

Primera: La reestructuración en los cuatro países considerados presentó modalidades o estilos muy distintos. La diferencia central radica en lo acaecido con los bienes de capital y de consumo duraderos, precisamente en los sectores en que predominan los capitales extranjeros. A grandes rasgos, el argumento es que en Chile se procedió a una desindustrialización, con fuerte reespecialización "hacia afuera" (ex-

$\square$ Los autores agradecen a los colegas Alejandro Vera Vassalle, João Carlos Ferrae, Jorge Katz y Wilson Peres por sus valiosos comentarios. Las opiniones expresadas en este artículo pueden no coincidir con las de la Organización.

1 Este artículo se escribió con anterioridad a la crisis que afectó a México en diciembre de 1994 y en su análisis considera las tendencias observadas en las economías sólo hasta 1993. Esta circunstancia afecta también al Brasil, porque se dejan fuera del estudio los posibles efectos del Plan Cardoso (julio 1994) sobre la competitividad internacional del sector manufacturero, en particular el efecto de la evolución del tipo de cambio.

2 Desde un punto de vista analítico, las ETs se singularizan en el conjunto de las empresas industriales latinoamericanas sobre todo por dos características. Primero, por su ubicación sectorial (ramas de mayor intensidad tecnológica y mayor dinamismo en la economía mundial); y, segundo, por la "agilidad" con que, como reacción a fuertes cambios en los entornos macroeconómicos e institucionales, tienden a abandonar o a reducir sus actividades productivas, reincorporarse a ellas, y cambiar sus funciones de producción. plosión importadora y exportadora); en Argentina se incurrió en una racionalización y una reespecialización que implicaron una "desofisticación" del parque manufacturero, e incluyeron una fuerte expansión de las importaciones (todo eso también significó desindustrialización, de modo que el término "desofisticación" se utiliza aquí para diferenciar el caso argentino del chileno, en función del tamaño y mayor complejidad de la industria sobreviviente en Argentina); en México hubo una reestructuración mediante una integración radical "hacia el Norte"; y en Brasil, el ajuste ha sido hasta el momento más bien "defensivo", tendiéndose a preservar la composición del parque productivo heredado del modelo sustitutivo (aunque con alguna pérdida de densidad tecnológica).

Segunda: Las empresas transnacionales protagonizaron, en los cuatro países estudiados, parte importante de las transformaciones que definieron los cuatro estilos distintos de reestructuración de la industria manufacturera después de la crisis del modelo de sustitución. Su comportamiento fue distinto en los cuatro países, lo que determinó la diferenciación de los cuatro estilos.

Tercera: La importancia relativa de las ETs es identificable en todos los casos por su ubicación sectorial. Su papel fue protagónico en la conformación de esos estilos por su actuación en los sectores en que participaron mayoritariamente en la industrialización sustitutiva (bienes de capital, bienes de consumo duraderos "modernos", y química/farmacéutica) y cuya adaptación a los nuevos tiempos estaba provocando fuertes cambios, no siempre favorables. Su rol fue coadyuvante en el refuerzo de importantes cambios ocurridos en las industrias de alimentos y en las ramas de bienes intermedios, productoras de commodities (química básica/petroquímica, metalurgia/siderurgia, papel y celulosa), que en general tuvieron fuerte expansión. Y su participación fue casi siempre marginal en los cambios (frecuentemente desfavorables) que experimentaron los sectores tradicionales (materiales para la construcción civil; textil; prendas de vestir; madera y muebles).

Cuarta: Los rasgos más generales de la influencia de las ETs en los cuatro estilos pueden captarse según la intensidad y forma con que ellas continuaron produciendo a través de sus plantas ubicadas en cada país. De forma esquemática, se puede sintetizar 
así los cuatro casos: en Chile, las ETs se destacaron por el abandono de la mayor parte de su anterior producción metalmecánica y electroelectrónica; en Argentina, por la disminución de sus actividades productivas en esas ramas, sea por abandono, sea por fuerte incremento del coeficiente importado; en México, por su actuación como agentes claves de la integración entre este país y los Estados Unidos, ampliando o reduciendo el tamaño de esas ramas, y elevando fuertemente los coeficientes de importación y exportación; y, en Brasil, por la decisión estratégica básica de ajustarse de manera de mantener su amplia presencia productiva en el gran mercado local de dichas ramas, implicando un menor coeficiente de apertura externa que en los demás casos.

Vale hacer notar que también en los sectores en que el papel de las ETs era más bien "coadyuvante" se advierten diferencias importantes entre los países. Cabe referirse, primero, a la distinta intensidad y grado de diversificación sectorial con que en cada país se desarrollaron la química y la petroquímica, la celulosa, la siderurgia, la metalurgia básica, y los alimentos no tradicionales; $y$, segundo, a la distinta intensidad con que las ETs participaron en el desarrollo de esas ramas productivas en cada país.

Quinta: Cada estilo de reacción de las ETs y a la vez cada estilo de reestructuración industrial fue el resultado de la combinación de tres conjuntos de factores internos de cada país: estructurales, macroeconómicos e institucionales.

Es importante señalar de partida que con la tesis de los cuatro estilos no se está negando otro elemento central de la evolución reciente de la industria manufacturera en América Latina como un todo, o sea, la existencia de algunos rasgos básicos comunes a los principales países de la región, vale decir, un relativo debilitamiento de los complejos industriales metalmecánicos, electroelectrónicos, y textiles, y un fortalecimiento también relativo de las ramas basadas en recursos naturales (Katz, 1994); bajas inversiones físicas y fuerte racionalización productiva; y elevado incremento de los coeficientes de exportación e importación. Más allá de tales semejanzas, las especificidades con que las tendencias señaladas se desarrollaron en cada país, han sido tan marcadas en cuanto a intensidad, momentos y velocidades, perspectivas futuras y determinantes, que se justifica caracterizar los cuatro casos como "estilos" de ajuste perfectamente diferenciados.

El hecho de que ya hayan transcurrido más de doce años desde el término del crecimiento por sustitución de importaciones nos abre la posibilidad de adoptar el método estructuralista para examinar los cambios que se han operado en cada país desde en- tonces. Dentro del enfoque estructuralista estamos priorizando en este texto, el análisis de los procesos de cambio en la composición y en el grado de modernidad de los sistemas productivos, así como en sus estilos de inserción internacional.

No cabe duda que el terreno para dicho análisis es muy fértil. Por un lado, no han faltado en América Latina, en el transcurso de la ultima década y media a dos décadas, grandes cambios en esas dimensiones económicas a las cuales se inclina el enfoque estructuralista. Esos cambios configuran una reorientación profunda de los estilos de comportamiento de las economías y de las modalidades de desarrollo de sus sistemas manufactureros. Por otro lado, no es difícil encontrar factores históricos determinantes de tal reorientación: se trata de un período de persistente turbulencia macroeconómica, y de toda una revolución en el marco regulatorio de las economías de la región. El período contiene un tercer ingrediente catalizador de las transformaciones, o sea, los elementos que provienen de la economía mundial: intenso progreso técnico y tendencia a la regionalización y a la globalización.

Este artículo se nutre en dos conjuntos de fuentes. En primer lugar se beneficia del hecho de que, contrariamente a lo que sucedía hace algunos años, en varios países de América Latina están surgiendo a nivel individual trabajos de investigación que proporcionan una visión de conjunto sobre la transformación radical que ha experimentado la industria manufacturera tras los efectos de la crisis económica y del proceso de apertura. A veces, este nuevo conocimiento cubre en forma satisfactoria el rol de las empresas transnacionales. ${ }^{3}$

En segundo lugar, se utiliza, por primera vez, el Programa de Análisis de la Dinámica Industrial (PADI), un programa elaborado por la División de Desarrollo Productivo y Empresarial de la CEPAL para fines de análisis de la industria latinoamericana. Frente a la escasez de datos de cobertura regional, los datos del

\footnotetext{
3 A nivel de cada país, la bibliografía más reciente sobre la industria manufacturera en su conjunto, y sobre las empresas transnacionales manufactureras en particular, incluye los siguientes textos: a) Argentina: textos de la compilación organizada por Kosacoff (1993), en especial los capítulos I y V; Aspiazu (en prensa), Katz (1994); CEPAL (1993c); Chudnovski, López y Porta, 1993 y 1994, y Beccaria y Kosacoff (cons.) (1994). b) Brasil: el informe final de un proyecto sobre competitividad de la industria brasileña, organizado por IEI/UNICAMP (1993); Fritsch y Franco (1991); CEPAL (1993 a) y b); Erber y Vermulm (1992); Barros (1993); Coutinho y Ferraz (1994); y Suzigan (1991). c) Chile: Rozas (cons.) (1992); Díaz, (1994); Agacino, Rivas y Román (1992); Castillo, Dini y Maggi (1994); Calderón (1994 b); y Aguilera y Becar (1991). d) México: Casar y otros (1989); Casar (1993 y 1994); CEPAL (1991); CET (1992); Peres (1990); Unger, Saldaña, Jasso y Durand (1992); Dussel Peters (1993); y Ros (1991).
} 
PADI representan un importante instrumento nuevo de análisis de los cambios estructurales en la industria manufacturera de América Latina.

El artículo se divide en cuatro secciones. La primera, detalla los instrumentos de análisis utilizados; la segunda, presenta una comparación esquemática de las cuatro sendas de ajuste, a base de una serie de indicadores de los cambios en la estructura y en la inserción internacional de las industrias manufactureras de los cuatro países; la tercera, contiene el análisis de los casos de los cuatro países seleccionados; y por último, a modo de conclusión, se aporta una visión conjunta de los cambios.

Caben, a esta altura, tres precisiones introductorias:

En primer término, se está obviamente tratando en este artículo una etapa muy difícil para la industria manufacturera de los cuatro países. Tras décadas de crecimiento acelerado, la producción manufacturera de estos países entró en un largo período de recesión, a partir de los años setenta en Argentina y Chile, y del inicio de los años ochenta, en Brasil y México. A excepción de Chile, su recuperación posterior sólo logrará aproximadamente el nivel absoluto de producción alcanzado antes de la crisis. Tomando como base el último año de crecimiento anterior a la crisis en cada país (Chile, 1972=100; Argentina, 1977=100; Brasil, 1980=100; y México, 1981=100) los índices de producto físico en 1993 fueron 140 en Chile, 96 en Argentina, 100 en Brasil y 114 en México.

En segundo término, el énfasis del artículo se concentra en el comportamiento de las subsidiarias de empresas extranjeras que se instalaron durante el proceso sustitutivo, o sea, que ya existían antes de la crisis económica de la región. Es necesario subrayar que durante los años ochenta los flujos de capital extranjero directo al sector manufacturero estaban esencialmente vinculados a esas empresas, es decir, eran flujos asociados a la preservación y a la amplia-

CUADRO 1

Flujos de inversión extranjera directa, 1977-1992

(1)

Flujos de IED (millones de dólares de 1992; promedios anuales)

Argentina ${ }^{a}$

1977-1980

1981-1985

1986-1989

1990-1992

Brasil

1976-1980

1981-1985

$1986-1990$

1991-1992

Chile

1976-1980

1981-1985

1986-1990

1991-1992

México

1976-1980

1981-1985

1986-1990

1991-1992

Total

1976-1980 5530

1981-1985 4236

1986-1990

1991-1992

537
565
966
3316



3354
1899
2508
1420

257

\section{4}

839

973

1202

1428

3416

6382

2091
(2)

Flujos de IED en

el sector manufacturero

(millones de dólares de 1992; promedios anuales)
(3)

(2)/ (1)

en $\%$
(4)

(1) como \% de la IED mundial

Fuente: Unidad Conjunta CEPAL/UNCTAD de empresas Transnacionales, a base de fuentes nacionales.

a Basado en cifras del Fondo Monetario Internacional. 
ción de sus actividades en la región. Las inversiones extranjeras a través de empresas totalmente nuevas estaban muy concentradas en los sectores de recursos naturales y de servicios - y eran el resultado de procesos de apertura de esos sectores al capital extranjero, casi siempre apoyados por procesos de privatización. ${ }^{4}$

En tercer y último término, además de esa orientación predominante de la inversión extranjera directa (IED, no financiera) hacia los recursos naturales y los servicios, destacan los siguientes hechos, que se presentan en forma esquemática, en la evolución del ingreso de la IED en los cuatro países considerados desde inicios de la década de 1980 (cuadro 1).

- El conjunto de países perdió participación en los flujos mundiales de IED, sobre todo durante la fuerte expansión de 1985-1990; pero a partir de 1991 dicha participación volvió a crecer.

\section{II}

\section{Metodología}

En esta sección presentamos el corte sectorial adoptado en el análisis y un breve resumen del esquema analítico utilizado para describir los procesos de cambio estructural en la industria manufacturera de cada país considerado.

\section{La presencia de las ETs: participación protagónica, coadyuvante o marginal en los grupos de sectores}

Hemos dividido la industria manufacturera en tres grupos de sectores, según si la participación de las ETs haya sido protagónica, coadyuvante, o marginal. Este corte sectorial es particularmente funcional para el análisis que presentamos, por dos razones: primero, porque además de destacar el rol de las empresas transnacionales, sirve directamente para diferenciar grupos de sectores según otros tres criterios, como son la organización industrial, el progreso técnico y las categorías de uso; y, segundo, porque muestra el perfil general de los cambios estructurales, dado que el grupo de sectores en que el papel de las ETs fue coadyuvante creció en todos los países; el grupo donde ese rol fue protagó-

4 El tema se está investigando para varios países a través de un proyecto contratado por el Banco Interamericano de Desarrollo y coordinado por Manuel Agosín, de la Universidad de Chile.
- México y Chile se distinguieron de Argentina y Brasil por su capacidad de atraer crecientes montos de inversión extranjera directa (IED). A este respecto, es muy interesante señalar que México fue el único país que logró atraer IED en tiempos de crisis (mediados de los años ochenta) ya que Chile lo ha hecho en tiempos de franca recuperación económica. Argentina recién ha recuperado su capacidad de atraer inversiones (esencialmente vía esquemas de privatización, a sectores de servicios), igualmente en una fase de recuperación;

- Una parcela decreciente de las inversiones se ha dirigido al sector manufacturero (lamentablemente no hay estadísticas para Argentina). Sin embargo, los montos absolutos que se dirigieron a este sector en México crecieron mucho en la segunda mitad de los años ochenta, y continuaban altos al inicio de los años noventa.

nico, presentó tendencias variadas según los países y el grupo en que la participación de las ETs fue marginal experimentó, en general, una contracción.

a) Grupo de sectores en los cuales las ETs desarrollaron un papel protagónico:

Estos sectores están comprendidos en las agrupaciones de la Clasificación Industrial Internacional Uniforme de todas las Actividades Económicas (CIIU) que se indican entre paréntesis.

- Bienes de capital mecánicos (382)

- Electro-electrónica/instrumentos científicos $(383 / 385)$

- Material de transporte (384)

- Química fina (352)

- Caucho (355)

- Tabaco (314)

- Vidrio (362)

Por lo demás, las empresas transnacionales predominan en estos sectores en casi todos los países del mundo. En América Latina la presencia del capital extranjero es particularmente elevada en la mayoría de sus países; prueba de ello es que su participación en las ventas supera casi siempre el 50 por ciento. Este grupo incluye a los sectores generadores y/o difusores de progreso técnico (bienes de capital/electrónica/quí- 
mica fina), y a los sectores oligopólicos con diferenciación acelerada del producto y gran intensidad tecnológica (electrónica de consumo y automotriz). Estos sectores son también los de mayor globalización productiva. Las ETs poseen aquí un unique asset (Hymer, 1976, Dunning, 1973), o sea, el dominio tecnológico (asociado a economías de escala y especialización internacional) que las coloca en situación enormemente ventajosa sobre las eventuales empresas locales, garantizándoles el liderazgo. Las dos excepciones en el grupo, a este respecto, son las ramas de los productos del caucho (neumáticos) y del tabaco, ambos oligopolios internacionales que se mantienen muy concentrados.

b) Grupo de sectores en los cuales el rol de las ETS fue coadyuvante:

Estos sectores están contenidos en las agrupaciones de la CIIU que se indican entre paréntesis.

- Alimentos y bebidas (311-3)

- Celulosa y papel (341)

- Química básica/petroquímica, excepto combustibles $(351,354,356)$

- Siderurgia/metalurgia básica (371/372)

Estos son esencialmente los sectores productores de bienes intermedios, los commodities industriales de uso difundido. El término coadyuvante tiene aquí dos sentidos, pues denota, por una parte que las empresas transnacionales tuvieron una participación importante pero no predominante en los mercados locales; y por otra, que estuvieron frecuentemente asociadas a empresas de capital nacional.

En el grupo de los alimentos y bebidas, la importancia de la presencia de las ETs es muy variada. Hay un conjunto de ramas en que la participación de estas empresas varió entre marginal y coadyuvante (justamente en los commodities, o sea, los alimentos tradicionales, semimanufacturados); y hay otro conjunto donde dicha participación fluctuó entre coadyuvante y protagónico (alimentos procesados, con alguna diferenciación de producto, y en general destinados a la venta en las cadenas de supermercados).

c) Grupo de sectores en los cuales el rol de las ETs fue marginal

Estos sectores están contenidos en las agrupaciones de la CIIU que se indican entre paréntesis.

- Textil/prendas de vestir/cuero y calzados (321 a 327)

- Madera y muebles (331/332)

- Imprentas (342)

- Minerales no metálicos, excepto vidrio (361/369)

- Productos metálicos (381)
- Otros productos manufactureros (390)

En estos sectores la participación de las empresas extranjeras tuvo escasa importancia en el desempeño productivo y tecnológico. Son sectores de tipo oligopólico competitivo, consumidores de tecnología, donde la competencia de precios es muy importante. Se observa en ellos una marcada heterogeneidad en cuanto a tamaño y capacidad tecnológica, destacándose la coexistencia de algunas empresas líderes (la mayoría de capital nacional) con numerosas empresas de tamaño mediano y pequeño.

\section{Esquema analítico: los cambios examinados y sus factores determinantes}

El gráfico 1 presenta el esquema de análisis utilizado. El objetivo es examinar los cambios de estructura y de inserción internacional ocurridos en la industria entre el final de la etapa de sustitución de importaciones y los primeros años de la década de 1990.

El análisis se compone de tres elementos. En primer lugar se identifican los cambios estructurales que se miden por tres conjuntos de indicadores: la composición sectorial; la inserción internacional, y la evolución de la productividad del trabajo.

En segundo lugar se consideran los mecanismos a través de los cuales se verifican los cambios en la composición del producto y de la productividad. Esos mecanismos pueden dividirse en inversiones/desinversiones físicas; racionalización pura y simple; y racionalización a través de la introducción de tecnologías desincorporadas. $\mathrm{Al}$ respecto es necesario aclarar que se presentan algunos problemas. Por un lado, no existen estadísticas relativas a la acumulación de capital fijo, no sólo a nivel de los distintos sectores y ramas, sino también a nivel de la industria manufacturera en su conjunto. Por otro lado, las informaciones disponibles sobre los procesos de racionalización son poco sistemáticas, salvo con raras y parciales excepciones.

El tercer elemento está representado por los factores determinantes de los cambios en la estructura industrial. En este sentido se consideran tres conjuntos básicos de factores: los macroeconómicos (tasa de crecimiento, estabilidad de precios, tasa de interés y tipo de cambio), los estructurales (tamaño del mercado interno, grado de desarrollo de la industria metalmecánica previo a la crisis de la deuda, importancia relativa del mercado regional y frontera de recursos naturales exportables en el mediano plazo) y los institucionales (cambios en el marco regulatorio, de la competencia y las políticas industriales). 
GRÁFICO 1

\section{Esquema analítico}

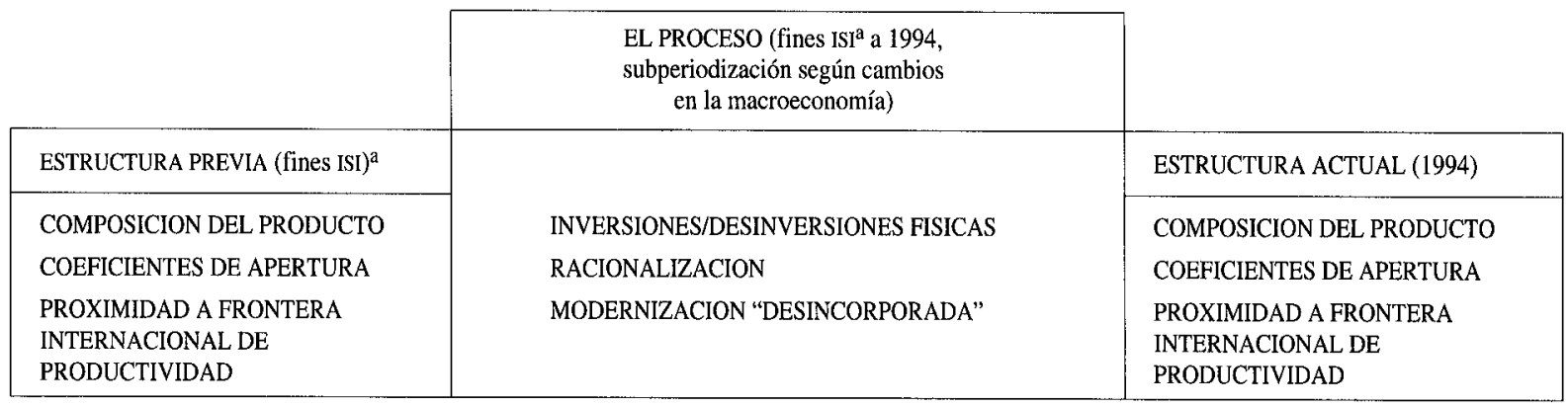

COMPORTAMIENTO DE LAS EMPRESAS TRANSNACIONALES

\begin{tabular}{|l|}
\hline DETERMINANTES ESTRUCTURALES \\
\hline Base productiva/distributiva \\
previa \\
Tamaño del mercado (interno \\
y regional) \\
Frontera de recursos \\
naturales
\end{tabular}

\begin{tabular}{|l|}
\hline DETERMINANTES \\
MACROECONOMICOS \\
\hline Crecimiento \\
Estabilidad de precios \\
Tasa de interés \\
Tipo de cambio \\
Velocidad de la apertura \\
\end{tabular}

DETERMINANTES INSTITUCIONALES Y EXTERNOS

Políticas

industriales/Organización

de mercados

Evolución tecnológica,

comercial y financiera

mundial

a ISI= Industrialización por sustitución de importaciones.

\section{III}

\section{Indicadores del cambio estructural}

Nos referiremos en esta sección a algunos indicadores relevantes de los principales cambios estructurales ocurridos en los países analizados, vale decir, el nivel y la composición del valor agregado; la evolución de la productividad del trabajo; y los cambios en la inserción internacional. 5

5 Los datos utilizados presentan algunos problemas - que hemos intentado solucionar, por lo menos en parte- derivados de la conversión (indispensable para la construcción de algunos indicadores) de los valores en moneda nacional a valores en dólares y a la determinación de valores reales para las variables consideradas. En el primer caso hemos utilizado las tasas de cambio del Fondo Monetario Internacional del tipo "rf", es decir, la tasa de cambio media anual; sin embargo no se han corregido las sobrevaluaciones/subevaluaciones de las monedas nacionales que eventualmente pueden haberse presentado en los años considerados. Esto significa que pueden haber algunas distorsiones (esencialmente en el caso de Argentina) de los coeficientes de exportación e importación, las que, de cualquier manera, no alteran las tendencias que se analizan a continuación. El segundo problema, en cambio, ha sido resuelto utilizando deflactores industriales para convertir los valores corrientes de valor agregado ( $\mathrm{y}$, consecuentemente, de productividad) a valores constantes.

\section{Nivel y composición del valor agregado}

En el cuadro 2 hemos resumido los resultados obtenidos en cuanto al nivel y la composición del valor agregado. Destaca en primer lugar la evolución de los sectores en los cuales el rol de las empresas transnacionales fue protagónico ("P") en los cuatro países.

En el caso de Chile se observa una reducción muy fuerte del peso de esos sectores "P" en la estructura industrial (de $30.7 \%$ en 1972 a $22.5 \%$ en 1992); también en el caso de Argentina se advierte una reducción, aunque de una intensidad menor. Por el contrario en México y, en menor medida, en Brasil, la importancia de los sectores "P" aumenta.

Si observamos más detenidamente ese mismo grupo de sectores, verificamos que en todos los países las ramas de bienes de capital se contrajeron, especialmente en Chile y Argentina, y que el caso de Brasil sigue diferenciándose de los otros tres por su mayor tamaño relativo $(17,3 \%$ del total industrial, con- 
CUADRO 2

Chile, Argentina, México, Brasil y OCDE: Nivel y composición del valor agregado (Porcentajes)

\begin{tabular}{|c|c|c|c|c|c|c|c|c|c|c|}
\hline \multirow[b]{2}{*}{$\begin{array}{l}\text { COMPOSICION DEL } \\
\text { VALOR AGREGADO }\end{array}$} & \multicolumn{2}{|c|}{ Chile } & \multicolumn{2}{|c|}{ Argentina } & \multicolumn{2}{|c|}{ México } & \multicolumn{2}{|c|}{ Brasil } & \multicolumn{2}{|c|}{ OCDE } \\
\hline & 1972 & 1992 & 1977 & 1992 & 1981 & 1992 & 1980 & 1993 & 1975 & 1992 \\
\hline \multicolumn{11}{|l|}{$\begin{array}{l}\text { Sectores con rol } \\
\text { protagónico de } \mathrm{ETs}^{\mathrm{a}}\end{array}$} \\
\hline $\begin{array}{l}\text { Metalmecánica/Electro- } \\
\text { electrónica }\end{array}$ & 9.7 & 5.1 & 13.6 & 7.7 & 10.4 & 8.8 & 18.7 & 17.3 & 22.8 & 25.3 \\
\hline Equipos de transporte & 8.0 & 2.4 & 10.8 & 12.5 & 7.9 & 9.6 & 7.8 & 8.3 & 10.7 & 11.5 \\
\hline Otros & 13.0 & 15.9 & 9.8 & 9.6 & 10.3 & 13.3 & 8.5 & 11.1 & 7.9 & 9.7 \\
\hline Subtotal & 30.7 & 22.5 & 34.2 & 29.8 & 28.6 & 31.7 & 35.1 & 36.6 & 41.4 & 46.5 \\
\hline \multicolumn{11}{|l|}{$\begin{array}{l}\text { Sectores con rol } \\
\text { coadyuvante de ETs }{ }^{\mathrm{a}}\end{array}$} \\
\hline Alimentos y bebidas & 21.3 & 28.4 & 18.4 & 25.7 & 23.2 & 23.1 & 13.6 & 14.9 & 11.5 & 11.1 \\
\hline Commodities & 14.4 & 20.7 & 14.1 & 15.0 & 16.7 & 20.1 & 19.9 & 23.6 & 18.9 & 17.4 \\
\hline Subtotal & 35.7 & 49.1 & 32.5 & 40.7 & 39.9 & 43.2 & 33.5 & 38.5 & 30.4 & 28.5 \\
\hline $\begin{array}{l}\text { Sectores con rol marginal } \\
\text { de ETs }\end{array}$ & 33.6 & 27.4 & 33.4 & 29.5 & 31.6 & 25.2 & 31.4 & 24.9 & 28.4 & 25.0 \\
\hline Total & 100 & 100 & 100 & 100 & 100 & 100 & 100 & 100 & 100 & 100 \\
\hline $\begin{array}{l}\text { Variación valor agregado } \\
\mathrm{ICE}^{\mathrm{b}}\end{array}$ & \multicolumn{2}{|c|}{0.41} & \multicolumn{2}{|c|}{0.33} & \multicolumn{2}{|c|}{0.28} & 100 & 100 & 100 & \\
\hline
\end{tabular}

Fuente: Programa PADI y elaboración propia.

a Véase la descripción de estos sectores en la sección II, "Metodología" (pp. 143-144) de este artículo.

b Indice cambio estructural.

tra $8.8 \%$ en México, $7.7 \%$ en Argentina y $5.1 \%$ en Chile). Comprobamos también que hubo una fuerte contracción de la industria automotriz chilena, lo que contrasta con la expansión de esa rama en los demás países, particularmente en Argentina y México.

En segundo lugar, llama la atención, en el cambio de la estructura industrial, el aumento de la importancia de los sectores en los cuales el rol de las ETs fue de tipo "coadyuvante" ("C"). Se incluyen aquí los commodities industriales (bienes intermedios) y los alimentos. ${ }^{6}$ El incremento del peso relativo de esos sectores en la producción industrial total fue especialmente fuerte en el caso de Chile (de $35.7 \%$ en 1972 a $49.1 \%$ en 1992); importante, pero de menor intensidad y más concentrado en los alimentos, en Argentina; ${ }^{7}$ y menor concentrado en los commodities,

6 En los alimentos, a pesar de la heterogeneidad de la agrupación 311 de la CIIU, están presentes varios productos, como la harina de pescado y los aceites vegetales, que son claramente insumos intermedios.

7 En realidad, los datos de Argentina para 1992 relativos a los commodities están sesgados por el hecho de que en ese año dejó en México y Brasil. En cambio, los sectores en que el rol de las ETs fue marginal muestran en todos los casos una reducción de su importancia relativa.

Una forma alternativa y de uso frecuente para comparar la intensidad de los cambios experimentados en la estructura industrial en los distintos países consiste en calcular algún "índice de cambio estructural". Se ha utilizado aquí el índice de cambio estructural de la ONUDI, "que mide la intensidad de las variaciones en la composición del valor agregado industrial (ICE, en el cuadro 2). Cabe recordar que un índice elevado no significa necesariamente un cambio en la dirección de la profundización de la industrialización (casos recientes de Corea y Taiwán) sino que puede reflejar una reversión hacia ramas de uso intensivo de recursos naturales y de menor intensidad

momentáneamente de funcionar una de las principales productoras de acero (SOMISA). Por lo tanto, los datos (aún no disponibles) de 1993, nos harían acercar más al caso chileno que al de los otros dos países, en lo que se refiere al incremento de importancia relativa de los bienes intermedios. 
tecnológica relativa. Esa es exactamente la causa del valor más elevado para Chile y más bajo para Brasil, entre los cuatro casos que estamos examinando.

En el cuadro 2 podemos observar también la distancia que separa de la OCDE a los cuatro países considerados, en lo relativo a la estructura industrial. Si tomamos la media de esos países desarrollados como punto de referencia para la definición de una estructura industrial articulada y competitiva, es fácil notar que, en los cuatro casos analizados no sólo existe una apreciable distancia, sino que además ella tiende a incrementarse. Sin embargo, aquí también cabe subrayar que tanto la distancia absoluta como su aumento tienen diferentes intensidades, siendo mayor la de Chile y menor la de Brasil (con Argentina y México como casos intermedios).

\section{Productividad del trabajo}

El análisis de los datos de productividad del trabajo ${ }^{8}$ (cuadro 3) nos muestra situaciones bastante dispares.

CUADRO 3

Chile, Argentina, México y Brasil: Productividad del trabajo

(Porcentajes)

\begin{tabular}{|c|c|c|c|c|c|c|c|c|}
\hline \multirow[b]{2}{*}{$\begin{array}{l}\text { VARIACION DE } \\
\text { LA PRODUCTIVIDAD }\end{array}$} & \multicolumn{2}{|c|}{ Chile } & \multicolumn{2}{|c|}{ Argentina } & \multicolumn{2}{|c|}{ México } & \multicolumn{2}{|c|}{ Brasil } \\
\hline & 1972 & 1992 & 1977 & 1992 & 1981 & 1992 & 1980 & 1993 \\
\hline \multicolumn{9}{|l|}{$\begin{array}{l}\text { Sectores con rol } \\
\text { protagónico de } \mathrm{ETs}^{\mathrm{a}}\end{array}$} \\
\hline $\begin{array}{l}\text { Metalmecánica/Electro- } \\
\text { electrónica }\end{array}$ & 100 & 103.7 & 100 & 170.5 & 100 & 118.9 & 100 & 139.3 \\
\hline Equipos de transporte & 100 & 88.0 & 100 & 225.5 & 100 & 151.1 & 100 & 133.7 \\
\hline Otros & 100 & 145.3 & 100 & 188.4 & 100 & 156.4 & 100 & 152.4 \\
\hline Subtotal & 100 & 136.4 & 100 & 198.4 & 100 & 144.1 & 100 & 143.7 \\
\hline \multicolumn{9}{|l|}{$\begin{array}{l}\text { Sectores con rol } \\
\text { coadyuvante de ETs }\end{array}$} \\
\hline Alimentos y bebidas & 100 & 82.4 & 100 & 194.3 & 100 & 137.1 & 100 & 129.0 \\
\hline Commodities & 100 & 176.0 & 100 & 149.0 & 100 & 171.2 & 100 & 147.5 \\
\hline Subtotal & 100 & 106.5 & 100 & 174.7 & 100 & 150.7 & 100 & 138.5 \\
\hline $\begin{array}{l}\text { Sectores con rol } \\
\text { marginal de } \mathrm{ETs}^{\mathrm{a}}\end{array}$ & 100 & 97.0 & 100 & 156.2 & 100 & 117.1 & 100 & 116.5 \\
\hline Total & 100 & 110.8 & 100 & 174.8 & 100 & 139.8 & 100 & 136.5 \\
\hline Variación valor agregado & 100 & 141 & 100 & 95 & 100 & 115 & 100 & 100 \\
\hline
\end{tabular}

Fuente: Programa PADI y elaboración propia.

a Véase la descripción de estos sectores en la sección II, "Metodología", (pp. 143-144) de este artículo.

- En el caso de Chile, en un contexto de crecimiento del valor agregado, la productividad experimentó un pequeño aumento (apenas $11 \%$ en veinte años), pero con una gran heterogeneidad en las tasas de variación que registran los diferentes grupos de sectores. Se destaca, en particular, el incremento de productividad de los commodities: $(76 \%)$.

- Una situación diferente es la de Argentina, donde, en un contexto de contracción de la producción industrial, se registra un fuerte crecimiento de la productividad ( $74.8 \%$ en quince años, lo cual implica una tasa media anual de $3.8 \%$ ). Las diferencias entre los sectores son marcadas, aunque menores que las que se observaron para Chile.

- En los casos de México y Brasil las tasas de variación de la productividad son parecidas ( $39.8 \%$ en un caso y $36.5 \%$ en el otro). Sin embargo, esa similitud se da en contextos diferen-

8 La productividad del trabajo ha sido calculada como valor agregado por persona ocupada. 
tes, dado que en México se registra un incremento (moderado) de la producción, y se combina la racionalización productiva con fuertes inversiones en algunos sectores, mientras que Brasil exhibe estancamiento del valor agregado industrial y procesos de racionalización en grado mucho mayor que de inversiones.

\section{Cambios en la inserción internacional}

Los cambios en la inserción internacional de los cuatro países en estudio (resumidos en los cuadros 4 y 5) una vez más presentan a la vez algunos elementos en común y otros muy diferentes:

\section{a) Coeficientes de exportación y de importación}

En términos generales, se puede apreciar un incremento, en los cuatro países, tanto de los coeficientes de exportación (es decir la relación entre las exportaciones y el valor bruto de la producción) como de los de importación (relación entre las importaciones y el valor bruto de la producción).

En los coeficientes de exportación, y considerando la intensidad del aumento, el primer lugar lo ocupa Chile, cuyo coeficiente crece de $4.3 \%$ en 1970 a $17.1 \%$ en 1992; muy fuerte es también la variación de estos coeficientes en el caso de México (de 3.1\% a $10.9 \%$ ). Por el otro lado, se producen cambios mucho menores en el caso de Brasil y, sobre todo, de Argentina. ${ }^{9}$

Además, hay otras diferencias, por el lado de la orientación que tomó en cada país este aumento de la inserción internacional.

En el caso de Chile los alimentos y los commodities muestran los coeficientes de exportación más elevados. En particular el de los alimentos pasa de $4.3 \%$ en 1972 a $21.7 \%$ en 1992. En Argentina, el coeficiente más elevado es el de los alimentos, pero el incremento más fuerte se registra en los commodities. Por el contrario, en México, es el sector automotriz el que encabeza el proceso de expansión de las exportaciones. En este caso los sectores "P" pasan de un coeficiente de apenas $3.3 \%$ en 1980 a uno de $21.8 \%$ en 1992 y en particular el de la agrupación 384 de la CIIU (equipo de transporte) pasa de $4.92 \%$ en 1980 a 35.91\% en 1992.

En Brasil la principal variación se registra por el lado de los commodities, cuyo coeficiente de exportación pasa de $5.5 \%$ a $22.1 \%$ en los años considerados.

9 Como se señaló antes, en este último caso hay probablemente una subestimación del coeficiente como consecuencia de la sobreevaluación de la moneda nacional en 1992.
Junto con el incremento de los coeficientes de exportación se registra también un aumento de los de importación. Sin embargo, también en este caso, hay profundas diferencias entre los cuatro países estudiados.

En primer lugar está el caso de Argentina, en el cual es particularmente elevado el crecimiento de ese indicador: ${ }^{10}$ de $6.3 \%$ en 1978 a $16.7 \%$ en 1992. Después viene el caso de México, donde el incremento del indicador es menor, pero el valor final es más elevado: $28.6 \%$ en 1992. Chile y Brasil presentan crecimientos menores en el coeficiente de importación. En el primer caso, se pasa de valores que ya en 1970 eran los más altos (entre los países considerados), a otros que son aún más elevados en 1992. Por el contrario, Brasil presenta los valores más bajos para ese indicador; sin embargo, considerando que la apertura es reciente, es legítimo esperar un importante aumento de los coeficientes de importación después de 1992.

En resumen, hay dos casos - Chile y Méxicode fuerte aumento en los coeficientes de exportación, pero que siguen acompañados por coeficientes de importación bastante elevados. Al mismo tiempo, la Argentina presenta un débil incremento de los coeficientes de exportación junto con un aumento sustancial de los de importación. Por el otro lado, está la senda recorrida por Brasil: frente a un pequeño crecimiento de los coeficientes de importación, hay un aumento de los de exportación que, sin ser espectacular como los de Chile y México, es de todas maneras mayor que el de Argentina.

\section{b) Balance comercial}

Este conjunto de situaciones diferentes se refleja también en el balance comercial. Con la sola excepción de Brasil, todos los demás países considerados empeoraron su situación (cuadro 4). Chile pasó de un déficit de 641 millones de dólares en 1970 a uno de 4718 en 1992; Argentina pasó de un superávit en 1978 de 624 millones a un déficit de 6412 en 1992 y México de un déficit de 13508 millones en 1980 a otro de 26625 en 1992.

Como se puede observar fácilmente en el cuadro 4 , en todos estos casos el déficit está concentrado en los sectores "P". Por otro lado, Brasil logró mejorar su posición, elevando su superávit de 2857 millones de dólares en 1980 a 11822 en 1992. Además, en este caso se alcanzó, en 1992, un superávit también en los dos rubros que eran negativos en 1980: los sectores "P" y los commodities.

\footnotetext{
${ }^{10}$ Aquí también vale lo que se dijo para el coeficiente de exportación, es decir que si se hubiera corregido la sobrevaluación de la moneda nacional, probablemente el coeficiente sería aún más elevado.
} 
Chile, Argentina, México y Brasil: Indicadores del sector externo

\begin{tabular}{|c|c|c|c|c|c|c|c|c|c|}
\hline \multirow[b]{2}{*}{$\begin{array}{l}\text { COEFICIENTES DE } \\
\text { EXPORTACION }(\%)\end{array}$} & & \multicolumn{2}{|c|}{ Chile } & \multicolumn{2}{|c|}{ Argentina } & \multicolumn{2}{|c|}{ México } & \multicolumn{2}{|c|}{ Brasil } \\
\hline & & 1970 & 1992 & 1978 & 1992 & 1980 & 1992 & 1980 & 1992 \\
\hline $\begin{array}{l}\text { Sectores con rol } \\
\text { protagónico de } \mathrm{ETs}^{\mathrm{a}}\end{array}$ & & 1.7 & 7.6 & 4.4 & 4.9 & 3.3 & 21.8 & 7.9 & 15.0 \\
\hline $\begin{array}{l}\text { Alimentos } \\
\text { Commodities }\end{array}$ & & $\begin{array}{r}4.3 \\
13.9\end{array}$ & $\begin{array}{l}21.7 \\
28.7\end{array}$ & $\begin{array}{r}15.5 \\
6.2\end{array}$ & $\begin{array}{l}18.4 \\
10.4\end{array}$ & $\begin{array}{l}2.0 \\
6.2\end{array}$ & $\begin{array}{r}1.8 \\
13.0\end{array}$ & $\begin{array}{r}22.1 \\
5.5\end{array}$ & $\begin{array}{l}19.7 \\
22.1\end{array}$ \\
\hline $\begin{array}{l}\text { Sectores con rol } \\
\text { marginal de ETs }{ }^{\mathrm{a}}\end{array}$ & & 2.4 & 10.4 & 2.2 & 4.2 & 1.9 & 6.2 & 5.2 & 10.2 \\
\hline Total & 。 & 4.3 & 17.1 & 7.5 & 9.0 & 3.1 & 10.9 & 9.2 & 16.2 \\
\hline $\begin{array}{l}\text { COEFICIENTES DE } \\
\text { IMPORTACION }(\%)\end{array}$ & & & & & & & & & \\
\hline $\begin{array}{l}\text { Sectores con rol } \\
\text { protagónico de ETs }\end{array}$ & & 61.8 & 122.3 & 12.0 & 33.6 & 37.0 & 59.2 & 11.6 & 14.0 \\
\hline $\begin{array}{l}\text { Alimentos } \\
\text { Commodities }\end{array}$ & & $\begin{array}{l}10.0 \\
39.5\end{array}$ & $\begin{array}{r}5.9 \\
46.6\end{array}$ & $\begin{array}{r}1.2 \\
13.9\end{array}$ & $\begin{array}{r}2.9 \\
25.4\end{array}$ & $\begin{array}{r}4.5 \\
25.5\end{array}$ & $\begin{array}{r}7.4 \\
27.6\end{array}$ & $\begin{array}{r}1.9 \\
12.9\end{array}$ & $\begin{array}{r}5.2 \\
11.6\end{array}$ \\
\hline $\begin{array}{l}\text { Sectores con rol } \\
\text { marginal de ETs }\end{array}$ & & 11.4 & 24.2 & 1.8 & 7.3 & 4.9 & 16.1 & 1.3 & 4.2 \\
\hline Total & & 29.7 & 43.2 & 6.3 & 16.7 & 16.8 & 28.6 & 7.4 & 9.5 \\
\hline $\begin{array}{l}\text { BALANZA COMER } \\
\text { (millones de dólares) }\end{array}$ & & & & & & & & & \\
\hline $\begin{array}{l}\text { Sectores con rol } \\
\text { protagónico de } \mathrm{ETs}^{\mathrm{a}}\end{array}$ & & -439 & -4359 & -1095 & -7067 & -8484 & -16506 & -1834 & 613 \\
\hline $\begin{array}{l}\text { Alimentos } \\
\text { Commodities }\end{array}$ & & $\begin{array}{l}-36 \\
-93\end{array}$ & $\begin{array}{r}942 \\
-641\end{array}$ & $\begin{array}{r}1787 \\
-537\end{array}$ & $\begin{array}{r}3295 \\
-1863\end{array}$ & $\begin{array}{r}-716 \\
-3482\end{array}$ & $\begin{array}{l}-2420 \\
-4522\end{array}$ & $\begin{array}{r}5949 \\
-2925\end{array}$ & $\begin{array}{l}4038 \\
4472\end{array}$ \\
\hline $\begin{array}{l}\text { Sectores con rol } \\
\text { marginal de } \mathrm{ETs}^{\mathrm{a}}\end{array}$ & & -73 & -659 & 468 & -777 & -826 & -3177 & 1668 & 2699 \\
\hline Total & & -641 & -4718 & 624 & -6412 & -13508 & -26625 & 2857 & 11822 \\
\hline
\end{tabular}

Fuente: Programa PADI y elaboración propia.

a Véase la descripción de estos sectores en la sección II, "Metodología" (pp. 143-144) de este artículo.

c) Composición de las exportaciones y de las importaciones

El análisis de la composición del comercio (cuadro 5) nos presenta desde otro ángulo los elementos detectados hasta ahora.

Por el lado de las exportaciones, en el caso de Chile el elemento más relevante fue el claro desplazamiento de las exportaciones hacia los alimentos. En Argentina, mientras se confirmaba el peso muy relevante de los alimentos, se incrementaba de forma consistente la importancia de los commodities. En México, por el contrario, los sectores "P" representaron el principal rubro de las exportaciones industria- les; sin embargo, vale la pena reiterar que en buena medida esta reorientación de las exportaciones se explica esencialmente por el espectacular crecimiento del sector automotriz. Mientras tanto se redujo fuertemente el peso de los alimentos. En Brasil las exportaciones se desplazaron hacia los commodities $\mathrm{y}$, en menor medida, hacia los sectores " $\mathrm{P}$ ", mientras que se redujo considerablemente el peso de los alimentos.

Con relación a las importaciones, se destacan principalmente el fuerte peso de los sectores "P" (al comienzo y, aún más, al final de los períodos considerados) y una tendencia a la sustitución de importaciones (con la excepción de Chile) en los commodities. 
Chile, Argentina, México y Brasil: Composición de las exportaciones e importaciones (Porcentajes)

\begin{tabular}{|c|c|c|c|c|c|c|c|c|c|c|}
\hline \multirow[b]{2}{*}{$\begin{array}{l}\text { COMPOSICION DE } \\
\text { LAS EXPORTACIONES }\end{array}$} & \multicolumn{2}{|c|}{ Chile } & \multicolumn{2}{|c|}{ Argentina } & \multicolumn{2}{|c|}{ México } & \multicolumn{2}{|c|}{ Brasil } & \multicolumn{2}{|c|}{$\mathrm{OCDE}$} \\
\hline & 1970 & 1992 & 1978 & 1992 & 1980 & 1992 & 1980 & 1992 & 1980 & 1992 \\
\hline $\begin{array}{l}\text { Sectores con rol } \\
\text { protagónico de } \mathrm{ETS}^{\mathrm{a}}\end{array}$ & 11.3 & 9.3 & 16.6 & 16.6 & 27.7 & 58.5 & 26.1 & 31.7 & 48.6 & 58.9 \\
\hline $\begin{array}{l}\text { Alimentos } \\
\text { Commodities }\end{array}$ & $\begin{array}{l}25.0 \\
46.3\end{array}$ & $\begin{array}{l}41.6 \\
33.0\end{array}$ & $\begin{array}{l}51.5 \\
11.7\end{array}$ & $\begin{array}{l}52.5 \\
17.4\end{array}$ & $\begin{array}{l}18.7 \\
37.0\end{array}$ & $\begin{array}{r}4.8 \\
24.4\end{array}$ & $\begin{array}{l}44.1 \\
14.7\end{array}$ & $\begin{array}{l}19.2 \\
33.0\end{array}$ & $\begin{array}{r}7.1 \\
25.6\end{array}$ & $\begin{array}{r}6.0 \\
19.7\end{array}$ \\
\hline $\begin{array}{l}\text { Sectores con rol } \\
\text { marginal de ETs }\end{array}$ & 17.4 & 16.1 & 20.3 & 13.6 & 16.6 & 12.3 & 15.1 & 16.1 & 18.7 & 15.3 \\
\hline Total & 100 & 100 & 100 & 100 & 100 & 100 & 100 & 100 & 100 & 100 \\
\hline $\begin{array}{l}\text { COMPOSICION DE } \\
\text { LAS IMPORTACIONES }\end{array}$ & & & & & & & & & & \\
\hline $\begin{array}{l}\text { Sectores con rol } \\
\text { protagónico de ETs } \\
\text { Alimentos } \\
\text { Commodities }\end{array}$ & $\begin{array}{r}60.3 \\
8.4 \\
19.1\end{array}$ & $\begin{array}{r}59.4 \\
4.5 \\
21.3\end{array}$ & $\begin{array}{r}54.9 \\
4.6 \\
31.2\end{array}$ & $\begin{array}{r}59.8 \\
4.5 \\
22.8\end{array}$ & $\begin{array}{r}56.4 \\
7.7 \\
27.8\end{array}$ & $\begin{array}{r}60.7 \\
7.5 \\
19.8\end{array}$ & $\begin{array}{r}47.8 \\
4.8 \\
42.8\end{array}$ & $\begin{array}{r}50.5 \\
8.7 \\
29.5\end{array}$ & $\begin{array}{r}40.4 \\
8.0 \\
28.8\end{array}$ & $\begin{array}{r}52.4 \\
6.6 \\
21.7\end{array}$ \\
\hline $\begin{array}{l}\text { Sectores con rol } \\
\text { marginal de ETs }\end{array}$ & 12.2 & 14.8 & 9.3 & 12.9 & 8.0 & 12.1 & 4.7 & 11.3 & 22.8 & 19.3 \\
\hline Total & 100 & 100 & 100 & 100 & 100 & 100 & 100 & 100 & 100 & 100 \\
\hline
\end{tabular}

Fuente: Programa PADI y elaboración propia.

a Véase la descripción de estos sectores en la sección II, "Metodología", (pp. 143 144) de este artículo.

\section{IV}

\section{Las ETs en cuatro estilos de reestructuración industrial}

En esta sección se analiza a grandes rasgos el rol que tuvieron las ETs en los distintos procesos de reestructuración manufacturera que experimentaron los cuatro países seleccionados desde el fin de la etapa de industrialización por sustitución de importaciones.

Los cuatro casos se examinan según las hipótesis y la metodología definidas en las secciones anteriores. Vale repetir los puntos principales que organizan el análisis que sigue:

- Las empresas transnacionales tuvieron reacciones distintas según se tratara de Chile, Argentina, México o Brasil, adecuándose a los entornos específicos donde actuaban, y contribuyeron en forma decisiva a que se generaran cuatro estilos de reestructuración;

- Dichas reacciones y estilos fueron el producto de la combinación de factores estructurales, macroeconómicos e institucionales específicos de cada país.

- No sólo los factores son distintos según el país que se examine, sino también las fechas en que ocurrieron los cambios a nivel estructural, macroeconómico e institucional, diferencias que han sido respetadas en el análisis. Las fechas en que se inicia el examen de cada país son diferentes, ya que corresponden al término del proceso de sustitución de importaciones específico de cada uno: 1973 para Chile, 1978 para Argentina, 1982 para México y 1981 para Brasil. También las reformas económicas, la apertura de las economías y la estabilización macroeconómica ocurrieron en fechas distintas según los países. Por 
todo lo anterior, el estadio de maduración de los cambios no es el mismo en cada país.

- La especificidad de la actuación de las ETs y su influencia en los "estilos" de reestructuración se captan por dos características: su ubicación sectorial, y la agilidad con que abandonan (parcial o totalmente) o se incorporan a la producción en cada país.

\section{Chile}

Los rasgos centrales de la reestructuración del sistema manufacturero chileno son: la desindustrialización protagonizada por las ETs en los años setenta, y la posterior reorientación "hacia afuera", mediante inversiones en ramas de uso intensivo de recursos naturales, en que las ETs participaron como actores coadyuvantes.

\section{a) Análisis de las tendencias}

Entre las economías latinoamericanas castigadas por la crisis de la deuda, la chilena fue la primera en salir de las dificultades macroeconómicas. Después de una década de bajo crecimiento, que culminó con grave crisis en los primeros años del decenio de 1980 , Chile reingresó a una senda de crecimiento con estabilidad. Entre 1984 y 1993 el PIB creció en un 7\% al año y el producto industrial en un $7,4 \%$.

i) Período 1973-1983. Las empresas transnacionales manufactureras tuvieron un papel determinante en la contracción del sistema industrial chileno durante la etapa que se extendió hasta mediados de los años ochenta. Los sectores en donde su presencia era predominante disminuyeron de manera importante su participación en el producto industrial, y quedaron a un nivel relativo que prácticamente no se recuperó en la fase de fuerte crecimiento posterior.

Los casos más dramáticos fueron los de las empresas que abandonaron rápidamente la producción local en los años setenta, y procedieron a dedicarse a la comercialización interna de bienes importados desde sus matrices o desde otras filiales de su red internacional. En esos casos la "reconversión" se resumió esencialmente en el desarrollo de actividades subsidiarias, como las de distribución, marketing y asistencia técnica. Las empresas de montaje de vehículos y las de productos electrónicos de consumo ejemplifican ese comportamiento. Entre otras empresas abandonaron o redujeron drásticamente la producción local la Fiat, Peugeot, Renault, Citroën, GM, Ford, Philips y la General Electric.
En otros casos, la reconversión fue más lenta y parcial, pero de igual manera implicó una fuerte reducción del valor agregado internamente, por el acentuado incremento de los coeficientes de importación y, en la mayoría de los casos, sin una contrapartida de aumento de las exportaciones.

Pese a ello, y al costo social que significó, varios expertos en la economía industrial chilena reconocen que la parcela sobreviviente de las empresas industriales en alguna medida se fortaleció durante esa fase. En este sentido, el período 1973-1983 habría implicado una racionalización de la producción, en que más allá de la desverticalización productiva se reorganizó el trabajo - a base de numerosos despidos y de un fuerte costo social- y también la base técnica y organizacional de las empresas (Díaz, 1994; Agacino, Díaz y Román, 1992; Castillo, Dini y Maggi, 1994).

ii) Período 1984-1992. A partir de mediados de los años ochenta y junto con la estabilidad macroeconómica y el acelerado crecimiento se desarrolló la fase que más bien se podría denominar "positiva". Tras la profunda recesión del período 1981-1983, se produjo una fuerte recuperación y expansión de la industria que había resistido el proceso de desindustrialización.

Considerando el comportamiento de las empresas en cuanto a expansión y modernización, los últimos diez años pueden dividirse en dos fases: la de recuperación, hasta fines de los años ochenta, y la de expansión, a partir de entonces.

La fase de recuperación parece haber tenido dos características básicas.

Primero, no se registraron en la mayoría de las ramas manufactureras chilenas reconversiones de plantas industriales con miras a la exportación, ni tampoco parecen haberse intregado las plantas locales con redes de producción globalizadas. Hubo en el período un fuerte crecimiento del empleo, con la consiguiente caída de la productividad media del trabajo.

Lo anterior indicaría que la industria chilena sobreviviente de la crisis no habría atravesado durante los años ochenta por un proceso de reestructuración importante. Las empresas parecen haberse caracterizado esencialmente por la utilización de la capacidad productiva que estuvo ociosa durante la fase anterior. Esta característica se aplica también a las empresas extranjeras. No hay indicaciones de que estas últimas (Nestlé, Good-Year, Ciba-Geigy, Roche, Dow Chemical, etc.) se hayan diferenciado de las empresas locales 
en su comportamiento básico frente a las inversiones y a la modernización, y también estuvieron muy pasivas.

Tal comportamiento no deja de sorprender, porque el crecimiento acelerado suele incrementar la productividad (ley de Verdoorn). El tema es polémico y queda abierto a nuevas investigaciones. Las dudas se fundamentan, por ejemplo, en evidencias de que el ajuste continuó en el sistema industrial durante esa fase, mediante mecanismos como la especialización a través de la subcontratación de materias primas y partes, y la externalización de los servicios de apoyo a las empresas (Díaz, 1994).

Segundo, cabe reiterar que el comportamiento de los sectores en lo referente a productividad ha sido muy dispar, y que en los commodities (sobre todo la celulosa y algunos alimentos) ésta se elevó de forma acentuada. A este incremento se asocia la intensa actividad inversionista, fuertemente apoyada por el mecanismo de conversión de deuda externa en inversiones (Rozas, cons., 1992).

En el nuevo perfil de producción que adquirió en los últimos veinte años el sector manufacturero, las plantas procesadoras de celulosa, y de harina de pescado, y las de conservas, alimentos congelados, etc., son las que más contribuyeron al "modelo exportador" chileno.

Las empresas extranjeras tuvieron una participación entre coadyuvante y marginal en ese proceso. En la celulosa, participaron como coadyuvantes la empresa conjunta Shell/Scott Paper, la suiza Attisholz y la Simpson Paper (asociada de forma minoritaria a la nacional CMPC). Ya en el sector de alimentos dicha participación era muy inferior, correspondiendo el liderazgo de la producción exportadora al capital local (la participación más relevante de las ETs en la actividad alimentaria estaba en la producción para el mercado doméstico, como son los casos de Nestlé, CocaCola, etc.). La escasa importancia de las inversiones manufactureras de las ETs contrasta con sus cuantiosas inversiones extranjeras en otros sectores, sobre todo en el cobre y las telecomunicaciones, las "estrellas" del proceso de privatizaciones y de conversión de la deuda externa chilena. Entre 1986 y 1992 apenas 223 de los 1800 millones de dólares de ingreso de inversiones extranjeras directas se dirigían al sector manufacturero.

En cuanto a la etapa actual, es posible que se esté produciendo una expansión gradual de las inversiones físicas en el sector manufacturero como un todo. La participación de las inversiones en maquinaria y equipos en el PIB creció de menos de 5\% a más del $9 \%$ entre 1988 y 1992, lo que puede significar que se están realizando inversiones en la modernización y expansión de la producción manufacturera.

La nueva etapa puede también significar cambios importantes en las funciones de producción. Las investigaciones más recientes respecto al tema dan señales positivas en esa dirección (por ejemplo, Castillo, Dini y Maggi, 1994). Entre ellas vale la pena mencionar una investigación realizada en el ámbito de la CEPAL, en la cual se encontró en un importante conjunto de empresas transnacionales un proceso de introducción de nuevas técnicas de organización, dirigidas a mejorar la productividad y la calidad (Calderón, 1994b).

\section{b) Determinantes}

En los párrafos anteriores ya quedan presentados los principales elementos determinantes de la evolución de la industria chilena y del comportamiento de las ETs.

Primero está el hecho de que el sistema industrial fue muy castigado entre mediados de los años setenta y mediados de los años ochenta por la conjunción de aspectos macroeconómicos negativos y la radical liberalización económica. Entre 1974 y 1980, además de un crecimiento económico apenas moderado y sobre todo inestable, el sector manufacturero se vio muy afectado por una liberalización comercial radical unida a una creciente apreciación cambiaria. Y, de ahí hasta 1983, sufrió las graves consecuencias de una aguda crisis financiera y de una profunda recesión, sólo atenuada por una depreciación cambiaria y por la reintroducción provisoria de mayores niveles arancelarios.

Segundo están los elementos estructurales. Chile tiene un mercado interno relativamente pequeño, $y$ además muy apartado geográficamente de los principales mercados consumidores mundiales. Esa circunstancia le ha impedido históricamente avanzar hacia un sector manufacturero con un grado elevado de complejidad. Aun así, en las condiciones proteccionistas que rigieron durante el proceso sustitutivo (interrumpido a principios de los años setenta), Chile había logrado formar un sector metalmecánico de alguna significación. Sin embargo, es posible sugerir que, en relación con los sectores manufactureros de los países aquí examinados, el de Chile era el más vulnerable frente a esquemas de liberalización. Enfrentado a tantos factores macroeconómicos negativos, le resultó imposible reconvertirse con menores pérdidas de valor agregado.

Ante la ausencia de políticas destinadas a fortalecer las eventuales ventajas comparativas dinámicas, $\mathrm{y}$ dados los elementos estructurales con que entonces 
contaba Chile, las oportunidades de éxito competitivo en el sector manufacturero se limitarían a las ventajas estáticas derivadas de sus abundantes recursos naturales y a las condiciones de competitividad típicas de las ramas tradicionales en lo que respecta al mercado doméstico (protegidas por costos de transporte, perecibilidad de productos, sistemas de distribución, preferencia de consumidores, etc.).

Vale hacer notar, por último, que a diferencia de lo que normalmente se supone, entre los determinantes de la evolución favorable de algunas de las ramas de mayor éxito exportador basadas en recursos naturales, como la celulosa, los productos de madera y algunos alimentos, está un conjunto de subsidios estatales. Por ejemplo, la reforestación que viabilizó la expansión de la producción de celulosa y de madera ha sido objeto de incentivos rurales dirigidos, y las inversiones en celulosa y en alimentos se han beneficiado de generosos esquemas de conversión de la deuda externa. Tales subsidios han significado un claro y no despreciable contraste con la orientación general "no intervencionista" supuestamente practicada en Chile desde 1973.

\section{Argentina}

En Argentina, tal como en los demás países analizados en este artículo, una mirada hacia el comportamiento de las ETs ayuda a visualizar la reorientación del sistema manufacturero después del término de la industrialización sustitutiva. Esa reorientación tiene dos rasgos centrales: el debilitamiento de los núcleos dinámicos del "modelo sustitutivo", y el fortalecimiento de las ramas que utilizan en forma intensiva los recursos naturales. Las ETs han sido los actores principales del primer movimiento, protagonizando la reducción de los complejos metalmecánico y electroelectrónico, sea por abandono del país, sea por reconversión radical de las funciones de producción hacia altos coeficientes importados. En lo que se refiere al segundo movimiento, su participación ha sido coadyuvante en la reestructuración, de forma asociada al capital local.

\section{a) Análisis de las tendencias ${ }^{11}$}

i) Período 1978-1990. La secuencia de los hechos económicos en Argentina es muy conocida. Al término de los años setenta el sector manufacturero

\footnotetext{
11 Varios de los elementos aquí presentados se encuentran en un trabajo de Kosacoff y Bezchinski (1993) basado en una investigación de aproximadamente sesenta empresas transnacionales manufactureras en Argentina.
}

estaba sometido a un profundo choque provocado por la política macroeconómica del ministro Martínez de Hoz (1978-1981). Coincidieron, entonces, una rápida liberalización comercial, una fuerte apreciación cambiaria y una aguda recesión.

Quedaron expuestas, por primera vez en forma drástica, las debilidades relativas de las ramas más sofisticadas del parque manufacturero argentino -metalmecánica y electroelectrónica - frente a la competencia internacional. Se había montado durante el proceso de sustitución de importaciones una industria con un grado de complejidad y verticalización bastante elevados, en relación con el tamaño de la economía. Son conocidos los logros de esa industria, y en especial de sus ETs, sobre todo en la capacidad de realizar importantes intentos de adaptación a bajas escalas de producción, gracias a los esfuerzos tecnológicos de la ingeniería local. Sin embargo, tales avances se mostrarían insuficientes para enfrentar las dificultades que se fueron acumulando a partir de fines de los años setenta (Katz, 1974 y 1994).

La larga crisis económica, que se extendió desde mediados de los años setenta hasta el año 1990, interrumpió los flujos de IED hacia Argentina, y provocó el cierre de algunas plantas importantes y el retiro o contracción de grandes ETs manufactureras. En la metalmecánica, abandonaron el país, por ejemplo, General Motors, Citroën, Fiat, Peugeot, DKW, MSD, Olivetti y Chrysler, y se redujeron buena parte de las demás, como Massey-Ferguson, John Deere, Torri, General Electric y Brown Boveri. Prácticamente desapareció el sector de electrónica de consumo tradicional, antes dominado por empresas nacionales, y en su lugar montaron un polo de ensamblaje en una zona especial de procesamiento, en Tierra del Fuego, con grandes ETs como Hitachi, Sony, Sanyo y Grundig. También se retiraron varias ETs del sector farmacéutico, como Squibb, Lilly, SKF, Upjohn y Abbott.

En la etapa inicial de esa fase se produjo un ajuste por la vía de una racionalización de la producción basada sobre todo en el despido masivo de trabajadores y se lograron importantes incrementos en la productividad del trabajo ( $30 \%$ entre 1975 y 1980). Después de 1981 la economía argentina reintrodujo varios controles a las importaciones, debido a los problemas de la deuda externa, lo que dio a los sectores menos competitivos alguna capacidad de sobrevivencia. Aunque de forma más gradual, y siempre con inversiones físicas muy deprimidas, y bajos niveles de IED, continuó desarrollándose durante los años ochenta un ajuste por racionalización, que fue más 
allá de la simple eliminación del sobreempleo e incluyó ajustes en las líneas de producción y la introducción de modernas tecnologías desincorporadas.

Sin embargo, la industria sufrió durante toda la década las consecuencias de una evolución extremadamente desfavorable de la macroeconomía. Faltaron, por lo tanto, las condiciones adecuadas para que las empresas pudieran hacer los cambios radicales necesarios para avanzar hacia prácticas productivas más modernas y compatibles con las exigencias internacionales de competitividad. Los rasgos idiosincrásicos de la industria argentina (en esencial bajas escalas de producción y falta de especialización a nivel de las empresas), y el hecho de que el ajuste se hiciera sin el reemplazo suficiente de los equipos anticuados, fueron barreras demasiado altas para el incremento de la competitividad exigido por el nuevo modelo de inserción internacional, lo que provocó una apreciable contracción de los sectores que utilizan la tecnología en forma más intensiva, notablemente aquéllos ocupados por empresas transnacionales.

Pero no a todas las ramas les fue mal. Tal como en otros países de América Latina, se pasaría a utilizar de forma más intensa los abundantes recursos naturales como base para la expansión de los bienes intermedios de tipo commodities. Aunque de forma subsidiaria a los grandes grupos de capital local, se observó un incremento de las empresas transnacionales en las actividades vinculadas con el aprovechamiento de las ventajas que otorga la abundancia de recursos naturales. A partir de la apertura de nuevas fronteras en el sector primario, las ETs han vigorizado sus actividades y ampliado sus inversiones, sobre todo en los complejos petroquímico y agroindustrial. En el complejo gasífero-petrolero se descubrió mucho petróleo y gas natural, y se generó toda una nueva base de asociaciones entre grupos locales y grandes ETs como Dupont, Bayer, Hoechst, Chemical y Dow Chemical.

ii) Periodo 1991-1993. A partir de 1991 se inició una nueva fase en que se conjugó un fuerte crecimiento económico (tasa de crecimiento anual promedio del PIB del orden del 7\%) con dos hechos muy problemáticos que, una vez más, se dieron de forma simultánea: una rápida apertura comercial y una fuerte apreciación cambiaria, ambos contemplados en la lógica del programa de estabilización (Plan Cavallo). En ese nuevo entorno, se observan las siguientes tendencias:

- Las ETs están recuperando su participación en la industria argentina. Hay indicios de que se in- crementan gradualmente las inversiones extranjeras en el sector manufacturero, notablemente en las industrias alimentaria y automotriz. En el caso de los alimentos han llegado recientemente al país varias de las grandes empresas mundiales (Nabisco, Parmalat, Cadbury, entre otras), para disputar el mercado doméstico con otras gigantes mundiales que nunca han salido de él (CPC, Nestlé, Swift, Cargill, Dreyfuss) y con la transnacional argentina Bunge y Born. En la industria automotriz, está invirtiendo Autolatina (Ford y Volkswagen) aunque todavía de forma moderada; la GM y la Chrysler están regresando; está entrando la Toyota, y se están instalando en el país fabricantes de autopartes extranjeros, incluso brasileños.

- Lo anterior no se ha producido hasta el momento por medio de inversiones físicas importantes, sino que por la vía de la reactivación y adaptación de las capacidades instaladas. Los mayores flujos de IED se han dirigido al sector de servicios (bancos, comercio, telecomunicaciones, aerolíneas) y al sector petrolero (Calderón, 1994a).

- Parte importante de las actividades del capital extranjero en la industria manufacturera pasó a regirse por una nueva dinámica de comportamiento. A diferencia de lo ocurrido en la fase de sustitución de importaciones, esta vez las inversiones y el proceso de reestructuración estaban definidos por las estrategias diseñadas a raiz de la apertura económica y del imperativo de la inserción competitiva en la economía mundial. Entre otras consecuencias de la nueva dinámica, está el fuerte incremento de los coeficientes de importación y exportación -mayor en los de importación- derivado de un comercio en que los flujos intrafirma son particularmente elevados.

Según Kosacoff (org. 1993), desde la apertura de 1991 las empresas en general están adoptando un modelo de producción y de comercialización de tipo "tripartito": en primer lugar está la producción intramuros, que se reduce; en segundo lugar, hay un mayor coeficiente de importaciones de insumos y partes; $y$, en tercer lugar, hay una mayor participación de bienes importados en las ventas totales de las empresas, en sustitución de su producción local. Se puede sugerir, a partir de este esquema, que las ETs son más activas en el down-sizing de la producción local que las empresas nacionales, porque buena parte de sus importaciones se hacen a nivel intrafirma, con todas las ventajas que eso puede significar para la corporación a nivel mundial. 
Es posible, por lo tanto, identificar cuatro conjuntos de ETs de acuerdo con los sectores de actividad y las estrategias que están aplicando (Kosacoff y Bezchinski, 1993).

Primero están las ETs que se insertaron en los sectores de servicios mencionados (en asociación con capitales locales y aprovechando las oportunidades de negocio que ofrecía el proceso de privatización). Lejos, ésa es la nueva IED más importante en Argentina en el período reciente, ${ }^{12}$ segundo, las ETs continúan siendo actores coadyuvantes importantes en las actividades manufactureras que hacen uso intensivo de recursos naturales, que tuvieron un gran impulso en la década anterior. Tercero, en los principales sectores donde las ETs desempeñan históricamente un rol protagónico, las empresas se están "globalizando", en particular a partir de esquemas de integración a la red internacional de producción y distribución de sus corporaciones a nivel internacional; y finalmente, está el resto de las ETs, cuyas estrategias no son de globalización $\longrightarrow$ o lo son de forma muy parcial-- pero igualmente tienden hacia una nueva función de producción, con incremento del coeficiente de importaciones y de la comercialización local de bienes importados.

\section{b) Determinantes}

Como lo evidencia el análisis precedente, la reestructuración industrial argentina quedó determinada por una combinación desfavorable de factores macroeconómicos y estructurales. Por el lado macroeconómico, ya se mencionó la experiencia de recesión con apertura entre 1978 y 1981 y de recesión con cierre de ahí hasta 1990, que contrajeron el producto industrial en un $25 \%$. Y la fase reciente de crecimiento, estabilización, apertura y apreciación cambiaria.

La apreciación cambiaria unida a la fuerte recuperación económica está resultando en una gran expansión de las importaciones y en grandes saldos negativos en el balance de pagos, concentrados en particular en las ramas metalmecánica y electroelectrónica.

Por el lado de los factores estructurales, no cabe duda de que el aspecto "negativo" de la reestructura-

\footnotetext{
12 Aunque no se trate de actividades industriales, se plantean aquí interrogantes sobre el efecto futuro de las privatizaciones sobre la productividad industrial, en función, por un lado, de cambios en la calidad y en el precio de los servicios y, por otro, de la inducción al desarrollo de la red de proveedores y subcontratistas en la industria manufacturera local.
}

ción argentina y del comportamiento de las ETs $-\mathrm{o}$ sea, la desindustrialización en los núcleos dinámicos de la industria sustitutiva- es en buena medida producto de la deficiente escala de producción y de la escasa modernidad del parque montado durante el período sustitutivo. El Mercosur se presenta como un nuevo factor estructurante, con posibles efectos potenciales positivos en esa misma industria metalmecánica, y en especial en la automotriz, atenuantes del proceso de desindustrialización. Parece estar determinando una interesante división del trabajo que puede permitir a las ETs reducir la pérdida de sus enormes costos no recuperables (sunk costs) gracias a las economías de escala propiciadas por el acceso preferencial al mercado brasileño.

Por el lado de los factores que afectan positivamente a la reestructuración industrial argentina, hay, como se sabe, un aumento en la utilización de los recursos naturales, propiciado por el complejo "petrolero-gasífero", por la industria de la soya, por los frigoríficos, por el procesamiento de jugo de frutas, etc. Se confirma de ese modo la excelencia argentina en cuanto a su histórica capacidad de utilizar de forma muy competitiva los abundantes recursos naturales de que dispone el país.

Por último, y por el lado de los cambios institucionales, el aspecto de mayor relevancia ha sido, sin duda, la forma radical en que se llevó a efecto la liberalización comercial, productiva y financiera, primero a fines de los años setenta y ahora desde principios de los años noventa. Pero, en contraste, hubo en cada uno de los dos períodos mencionados por lo menos un episodio intervencionista importante que dejaría marcas profundas en la composición industrial vigente en Argentina a mediados de los años noventa. Primero, se otorgó amplios subsidios a los commodities, en pleno auge liberal; y ahora, el sector automotriz sigue fuertemente protegido.

\section{México ${ }^{13}$}

De los cuatro casos examinados en este artículo, quizá sea el de México en el que se percibe casi instantáneamente la importancia de las ETs en la reestructuración industrial. Las ETs han sido los agentes centrales de la reconversión de la industria mexicana hacia la integración con los Estados Unidos, y han pasado a liderar el comercio entre los dos países, en gran medida a través de operaciones intrafirma.

\footnotetext{
${ }^{13}$ Escrito antes de que se desatara la crisis de diciembre de 1994.
} 
El proceso de reestructuración del sector manufacturero mexicano tiene varias características que lo singularizan entre los casos aquí examinados. Entre ellas destaca el hecho de que ese proceso ha sido uno de los elementos de una reconversión radical "hacia afuera". Es el resultado de la forma radical en que el país tuvo que ajustarse para enfrentar la crisis de la deuda externa.

\section{a) Análisis de las tendencias}

El ajuste macroeconómico mexicano se realizó en dos fases, 1982-1987 y 1987-1992, que correspondieron a dos etapas de la reestructuracion del sistema manufacturero.

En la primera fase (1982-1987) el ajuste significó, inicialmente, una importante depreciación cambiaria, una rebaja salarial considerable y una fuerte recesión. También signifícó, hasta 1985, el total control cuantitativo de las importaciones; $y$, desde entonces hasta 1987, un relajamiento progresivo de este control, cuyo impacto potencial negativo de corto plazo en el balance de pagos fue, sin embargo, contrarrestado por una depreciación cambiaria adicional, resultante de la caída de los precios del petróleo en 1985-1986. El conjunto de factores macroeconómicos determinó un extraordinario incremento en el coeficiente de exportaciones del sector manufacturero, que se lanzó hacia el exterior a una velocidad singular en la historia económica mundial. En auxilio a ese movimiento, hubo una política industrial para distintos sectores - automotriz, petroquímica, computadores, maquilas - en la cual el comercio exterior era el elemento clave. Como resultado, entre 1981 y 1987 el déficit comercial del sector manufacturero había bajado de 17 a 1.4 mil millones de dólares.

En la segunda fase (1987-1992) hubo un viraje importante de las políticas de obtención de divisas, cuyo énfasis se desplazó de la obtención de resultados comerciales favorables hacia la atracción de recursos financieros. En 1987, y en gran medida como resultado de la depreciación cambiaria, la economía mexicana había llegado a altas tasas de inflación. En diciembre de ese año se realizó el "Pacto de estabilización", con el cual se logró bajar drásticamente la inflación en 1988 (e interrumpir una onda de fuga de capitales). Parte del éxito se debió a una apreciación del tipo de cambio, que ha sido desde entonces creciente, y que ha funcionado como elemento de preservación de la estabilidad de precios. Todo eso dio lugar simultáneamente a una segunda fase de liberalización, la que determinó un fuerte deterioro en el balance comercial. El creciente déficit fue financiado en 1988 con reservas, en 1989 y 1990 con privatizaciones, y a partir de 1990 con masivas entradas espontáneas de capitales bajo el estímulo de las perspectivas del NAFTA y luego bajo la influencia de la reducción de las tasas de interés norteamericanas.

Gracias a la estabilización de los precios, la economía se recuperó a partir de 1988 , lo que dio al sector manufacturero nuevas energías y mayor capacidad para resistir la apertura comercial, simultánea a la apreciación cambiaria. Pero el resultado final sería muy dispar según los sectores que se considere. Esa capacidad desigual de resistir a la competencia internacional en el mercado interno, junto al hecho de que la vocación exportadora exhibida en la primera fase del ajuste - y confirmada en la segunda fase- fue igualmente muy dispar entre sectores, determinó que a principios de los años noventa el sector manufacturero mexicano tuviera una configuración distinta a la que presentaba antes de la crisis. En todo eso el capital extranjero tuvo un papel decisivo.

El ajuste mexicano produjo el fortalecimiento de una serie de ramas y un relativo debilitamiento de otras. Entre las primeras están esencialmente las seis "estrellas" del sector manufacturero mexicano, de intenso comercio internacional: las industrias automotriz, de equipos de telecomunicaciones y de informática, petroquímica, del cemento, del vidrio y maquila.

En sólo un caso, el del vidrio, predomina el capital nacional, a través de la empresa Vitro. Esta, sin embargo, ha pasado recientemente por una fuerte "transnacionalización".

En otras dos industrias el liderazgo de las ETs es absoluto: la automotriz: GM, Ford, Chrysler, Volkswagen y Nissan; y la de equipos de telecomunicaciones y de informática: Ericsson, ATT, NEC, Panasonic, Alcatel, IBM, Hewlett Packard, Motorola, entre otras.

En las demás, la participación de las ETs es elevada. En las industrias química-petroquímica: Dupont, Bayer, Hoechst, Montsanto; en la de cemento: existe un duopolio de CEMEX, (empresa nacional) que controla alrededor del $65 \%$ del mercado, y Cemento Apases (empresa transnacional) con el $35 \%$ del mercado; en la maquila: aproximadamente la mitad de las 2142 empresas maquiladoras son norteamericanas, y otro $10 \%$ son extranjeras de distintas nacionalidades.

Cabe observar que algunas de las mismas ra- 
mas que se desarrollaron en las maquilas, entre ellas la de electrónica de consumo y la de los electrodomésticos, se han reducido fuertemente en el resto de México, $y$, tal como en las zonas maquiladoras, tienden a ser ramas de ensamblaje. Las nuevas ETs que se establecieron en el país lo hicieron principalmente en las zonas de maquila (General Electric, Zenith, Sony, Hitachi), mientras que las preexistentes, nacionales o extranjeras, desaparecieron o transformaron en ensamblaje la producción predominantemente local por la vía de un fuerte incremento del coeficiente de importación. Por cierto que ese comportamiento contribuyó a bajar el grado de complejidad y de integración vertical de la industria mexicana. Desde ese punto de vista no se las puede llamar ramas "vencedoras".

Entre las ramas definitivamente "perdedoras", la mayoría son empresas locales (textil, calzados, alimentos, celulosa, metalurgia). El sector de bienes de capital en el que internacionalmente la presencia de las ETs es fuerte, nunca ha sido muy fuerte en México, y las ETs que se instalaron durante el proceso sustitutivo redujeron sus actividades productivas o sencillamente abandonaron la producción local.

La heterogeneidad de comportamientos fue resultado de la conjunción de los efectos de las dos fases del ajuste macroeconómico. En la fase en que la industria era castigada por la recesión y fomentada por las condiciones de "competitividad espuria" favorables (relación cambio-salario muy elevada y protección a las importaciones), los sectores "ganadores" se modernizaron y fortalecieron su integración con el mercado del Norte mediante la racionalización y la modernización, mientras que los demás sectores se quedaron estancados en las condiciones de la crisis. En el período siguiente, de rápida apertura económica, los "ganadores" ya habían tomado la senda del mejoramiento de la productividad y de la calidad, condiciones suficientes para seguir exportando y para aprovechar la mejora del mercado interno, aun en condiciones cada vez más desfavorables en materia de tipo de cambio; los "perdedores", en cambio, resentirían fuertemente las nuevas condiciones de apertura con apreciación cambiaria, a pesar del mejoramiento del mercado doméstico.

Pese a que en algunas ramas fracasó el capital extranjero, el balance final del desempeño de las ETs manufactureras es positivo, desde el punto de vista de la modernización y la inserción internacional. Cabe señalar que el desempeño del conjunto de las ramas en que la presencia de las ETs era masiva fue muy superior comparado con el del conjunto de las ramas en que esa presencia fue escasa.

Dado que los sectores con elevada participación del capital extranjero también eran los que presentaban mayor dinamismo, inserción internacional y progreso técnico, se puede concluir que el sistema industrial de México se movió en una dirección positiva, y que en ese movimiento el papel de las ETs fue decisivo.

Sin embargo, esta visión positiva debe complementarse con algunas reservas. La primera se refiere a la relativa debilidad del sector de bienes de capital (Casar y otros, 1989). Si bien el comportamiento de las distintas ramas de este sector también fue heterogéneo --por ejemplo, a la industria de equipos de telecomunicaciones le fue bien (Peres, 1990), contrastando con sectores como el de máquinas herramientas, al cual le fue mal (Unger, Saldaña, Jasso y Durand, 1992)—, lo cierto es que la reestructuración mexicana no fortaleció el parque productor de dichos bienes que es relativamente pequeño.

La segunda reserva tiene relación con la industria de bienes intermedios de intenso comercio internacional. También aquí la evolución ha sido muy heterogénea. De un lado está el éxito de la industria petroquímica, y del otro, la mucho menos favorable evolución de la mayoría de las demás ramas que utilizan intensivamente recursos naturales, lo que ha provocado fuertes déficit comerciales.

La principal inquietud con respecto a la experiencia de reconversión industrial en México radica en la insuficiente respuesta al problema del déficit del balance comercial que originó el proceso de ajuste en el país. Los desequilibrios externos son hoy superiores a los que originó dicho ajuste. En los años 1991, 1992 y 1993 resurgieron enormes déficit comerciales en la industria manufacturera (US\$ 26 mil millones en 1993, el doble del de 1980). El viraje de la política económica en 1987, en que se pasó a privilegiar la estabilización de precios por sobre los objetivos anteriores, dio excelentes resultados en el combate contra la inflación, en la entrada de capitales, y resultados razonables en la recuperación del crecimiento, pero también amplió la vulnerabilidad externa de la economía.

\section{b) Determinantes}

Junto a los determinantes macroeconómicos, comentados anteriormente, los factores estructurales también contribuyeron al estilo de reestructuración mexicano. Como elementos básicos podemos mencionar un mercado interno de proporciones razonables, y la 
vecindad con los Estados Unidos, que le permitió a la industria mexicana una integración fuerte y cada vez mayor con ese gran mercado. Además, la estructura productiva medianamente desarrollada que México tenía al momento en que irrumpió la crisis de la deuda, dio al sector manufacturero una capacidad razonable para enfrentar la recesión y la liberalización comercial sin sufrir el grado de desindustrialización observado en otros casos en América Latina. Por ultimo, México parece diferenciarse de los otros tres países considerados por disponer de fronteras agrícolas menos amplias. En términos generales, en comparación con los demás países examinados la producción y las exportaciones de bienes de uso intensivo de recursos naturales (salvo en la petroquímica) se expandieron relativamente menos en México.

Finalmente, cabe mencionar los determinantes "institucionales". El caso mexicano se menciona frecuentemente por el exitoso resultado de las reformas que tuvieron como eje el proceso de liberalización económica y desregulación estatal. Aún no queda claro hasta qué punto los éxitos fueron el resultado de la apertura comercial y otras reformas liberalizantes, porque no hay análisis detallados del impacto de la apertura y la desregulación en la industria manufacturera. Sin embargo, existe la sensación de que los casos de ajuste más exitosos se dieron justamente en aquellos sectores en que hubo intervención estatal y políticas industriales muy claras: las industrias automotriz, de computadores, petroquímica y maquilas.

\section{Brasil}

Frente a los otros tres casos analizados, la evolución del sector manufacturero brasileño se caracteriza porque el parque montado durante el proceso de sustitutición de importaciones preservó mucho más de lo que estaba estructurado al final de dicho proceso. En comparación con Chile y Argentina, preservó mucho más de la composición sectorial previamente establecida; en tanto en relación con México, su "reorientación hacia afuera" se dio en mucho menor escala.

El comportamiento de las ETs fue decisivo en esa evolución. En esencia, las ETs trataron de conservar sus importantes posiciones relativas en el mercado local y sus cuantiosas inversiones físicas previas. A diferencia de lo que hicieron en Chile y Argentina, en Brasil abandonaron sólo una mínima parte de la producción local, de modo que casi todas las grandes ETs que al final del proceso de sustitutición lideraban la producción local seguían haciéndolo a principios de los años noventa. Y, contrastando con su proceder en México, la toma de decisiones continuó en esencia asociada a la participación en el mercado interno. ${ }^{14}$

\section{a) Análisis de las tendencias}

En la década de 1980 las ETs contribuyeron no sólo a las tendencias negativas - baja inversión y atraso tecnológico relativo- sino también a las positivas, sobre todo las asociadas al incremento de las exportaciones. En los años noventa se están mostrando muy activas en el proceso de ajuste, reforzando una tendencia que parece estar apuntando hacia la preservación y la modernización del parque industrial brasileño.

En los años ochenta, caracterizados por una relativa "pasividad", destacaban tres aspectos básicos:

- El aumento de la participación relativa de los sectores de bienes intermedios en la producción $\mathrm{y}$, sobre todo, en las exportaciones industriales fue en gran parte consecuencia de una serie de inversiones destinadas originalmente al mercado interno. Estas inversiones frenaban parte de un ciclo inversor iniciado a partir de mediados de los años setenta y que maduró a comienzos de los años ochenta. El capital extranjero fue un socio muy activo en esas inversiones, y participó como protagonista en el sector del aluminio (Alcan, Alcoa y Billington/Shell), y como coadyuvante en el de la petroquímica (Rhone-Poulenc, Dow Chemical, Dupont, Bayer, Hoechst), la celulosa (Champion, Aracruz/British Tobacco, Cenibra/Mitsubishi) y la siderurgia (Mannesman, Belgo-Mineira, capitales japoneses en Tubarao y en Usiminas).

- El resto del sistema industrial - que en su mayor parte se había recién instalado o modernizado en los años setenta- quedó relativamente intacto durante los años ochenta.

La recesión, el bajo nivel de inversiones y el fuerte cierre de la economía explican básicamente ese comportamiento. La década se caracteriza por la ausencia de cambios importantes tanto en la composición de la capacidad productiva como en relación con su modernización. El comportamiento de las ETs en esa década puede caracterizarse por su relativa pasi-

\footnotetext{
${ }^{14}$ Este artículo se basa en informaciones y análisis anteriores al "Plan Cardoso", el plan de estabilización implantado en julio de 1994. Cuando lo dimos a divulgación, en noviembre de 1994 , no estábamos considerando la eventual reversión de las tendencias que podrían resultar de la probable preservación de la fuerte apreciación cambiaria que siguió a la implantación del Plan.
} 
vidad, pese al hecho de que, al igual que las empresas nacionales, incrementaron mucho sus exportaciones (CEPAL, 1993a). Un buen ejemplo de esa pasividad son las cifras relativas a la evolución de la productividad del trabajo, que muestran un virtual estancamiento entre 1980 y 1990 , incluso en los sectores de gran intensidad de capital extranjero.

Las mismas ETs que al final del proceso de sustitución lideraban la producción local se mantuvieron a la cabeza en toda la década de 1980. Con raras excepciones, permanecieron en una actitud de "esperar para ver". En la lista de las empresas líderes en 1980, que siguen a la cabeza en los años noventa, figura una parte importante de las grandes corporaciones mundiales de la metalmecánica y la electroelectrónica; e incluye, con un comportamiento algo menos pasivo, a las grandes corporaciones de la química fina.

En la industria automotriz figuran General Motors, Fiat, Ford, Volkswagen, Mercedes Benz, Volvo, Cummins, TRW, Bosch, Krupp, etc.; en la de equipos de transporte agrícola y de carga, aparecen Valmet, Massey-Ferguson, Ford, Caterpillar, etc.; en la de equipos eléctricos, están Siemens, Asea, Brown Broveri, mecánica pesada; entre los sistemas de telecomunicaciones, se cita a Ericcson, Equitel/Siemens, Alcatel, NEC.

En otros equipos pesados, Ishikawagima, Voith, $\mathrm{CBC}$; en la industria de electrodomésticos y de electrónica de consumo, Philips, Philco, Toshiba, Mitsubishi, Brastemp/Whirlpool; en informática, se incluyen IBM, Unysis y Xerox; y en la química fina, Roche, Ciba-Geigy, Bayer, Sandoz, etc.

- Hubo una fuerte caída en el valor de exportación de los alimentos como consecuencia de la evolución poco favorable del comercio mundial (proteccionismo europeo). Se redujeron en forma sustantiva los precios de las principales semimanufacturas alimentarias, como el café, la soya y el azúcar, y la caída no fue mayor sólo debido a la fuerte expansión de las exportaciones de productos no tradicionales, en especial el jugo de naranja. La participación de las ETs en el deterioro de esos precios fue bastante marginal, con excepción de empresas como la Cargill y la Bunge y Born. Las grandes empresas extranjeras en ese sector de Brasil (Nestlé, Sanbra/Bunge y Born, Coca-Cola) se dedicaban principalmente a la producción de alimentos destinados al mercado doméstico moderno - en los cuales, no parece que se produjeron inversiones físicas o cambios tecnológicos significativos.
La relativa pasividad de los años ochenta contrasta con los cambios importantes que se están produciendo desde 1990. El sector manufacturero brasileño está pasando por un ajuste significativo en el cual las ETs participan intensamente (CEPAL, 1993a y b). Las principales características del ajuste pueden resumirse como sigue:

i) Se trata de un proceso con carácter de urgencia para las empresas, determinado por la agudización de la crisis macroeconómica y, de forma complementaria, por la simultánea y rápida liberalización de la economía. Según varios estudios que se vienen realizando en Brasil, el viraje está ocurriendo también en las empresas nacionales (véase por ejemplo, IEI/UNICAMP, 1993).

ii) Se está racionalizando el proceso productivo, con miras a reducir los costos y mejorar la calidad, incluyendo mecanismos como la desverticalización, la especialización, la flexibilización y la introducción de nuevas técnicas de gestión, como los procedimientos de "calidad total", ISO 9000, just-in-time, etc. Lo novedoso no es tanto que estén ocurriendo esos cambios -en México y Argentina existen procesos semejantes desde mediados de los años ochenta- sino la velocidad e intensidad con que se verifican.

iii) El ajuste que afectó a las ETs en la industria brasileña desde 1990 está teniendo importantes consecuencias positivas en su competitividad. Si bien la recesión impide que el ajuste incluya grandes inversiones en nuevos equipos - de ahí que todavía no se haya avanzado mucho en la automatización industrial- estarían en proceso grandes mejoras en la productividad (que en el período 1991-1993 creció en $30 \%$ ), y la calidad.

iv) Los principales indicios apuntan a que el ajuste está dando impulso a las empresas brasileñas para sobrevivir en las actuales condiciones de retracción interna y de apertura externa. El patrimonio productivo formado durante la industrialización sustitutiva estaría en lo esencial siendo preservado y reconvertido.

v) Sin embargo, es importante destacar dos aspectos de esa supuesta capacidad de evitar que la apertura provoque la desindustrialización. En primer lugar, dicha apertura es demasiado reciente para que se pueda evaluar en forma adecuada sus efectos; y en segundo lugar, la ausencia de inversiones en capital fijo está señalando que muy pronto cesarán los incrementos de productividad. La conclusión sería que la competitividad de la industria brasileña depende fundamentalmente de que se retomen de forma sustentable el crecimiento y las inversiones, lo que a su vez depende del control de la inflación. 
vi) Se observan también, a partir de la apertura, el debilitamiento de ramas de intenso progreso técnico a nivel internacional, y con fuerte participación de ETs, como las de fármacos, componentes electrónicos y computadores. Otro caso de down-sizing parece ocurrir en el sector de bienes de capital en serie - en que las ETs en Brasil tienen una participación de tipo "coadyuvante".

\section{b) Determinantes}

Al igual que cada uno de los casos descritos, el brasileño tiene sus singularidades, resultantes de factores macroeconómicos, estructurales e institucionales muy específicos. Vale la pena describirlas mediante una comparación con los demás casos.

Comenzaremos por los factores macroeconómicos. En primer lugar, a diferencia de Argentina y México, Brasil no tuvo que enfrentarse, por lo menos hasta la reciente reforma monetaria (Plan Cardoso, de julio de 1994) a la difícil coyuntura de liberalización comercial con apreciación cambiaria. ${ }^{15}$ Claro está que el comportamiento futuro de las empresas, extranjeras y nacionales, dependerá de cómo evolucione el tipo de cambio. En segundo lugar, y no menos importante, Brasil es el único de los cuatro países considerados que a mediados de 1994 todavía no había logrado alcanzar una estabilidad de precios. Eso explica por qué, a diferencia de México, el importante ajuste microeconómico que experimentan las empresas $-\mathrm{y}$ las ETs- no incluye inversiones fijas de montos significativos. La consecuencia obvia es que la competitividad a largo plazo sigue comprometida por la flagrante insuficiencia de inversiones físicas.

En tercer lugar, la crisis de la deuda afectó con mucho menor intensidad a las empresas en Brasil que en los otros países. El ajuste financiero se dio luego del inicio de la crisis, y sin quiebres (1981-1983), y la situación financiera de las empresas se consolidó positivamente gracias a tres buenos años para sus negocios (1984-1986). En cuarto lugar, sus problemas de balance de pagos fueron mucho menores que los de México, Argentina y Chile -lo que se manifestó muy tempranamente, ya a partir del segundo semestre de 1983, cuando surgieron grandes saldos comerciales. De modo que, a diferencia de México, por ejemplo, Brasil no sufrió la exigencia macroeconómica de reorientar radicalmente el sistema productivo hacia el exterior en los años ochenta.

En cuanto a los factores estructurales, Brasil pre-

15 Véase la nota 13. senta dos diferencias básicas con relación a Chile y Argentina - $y$, de algún modo, también a México-. La primera es que la reestructuración industrial brasileña partió de una base industrial más compleja e integrada, $\mathrm{y}$, en consecuencia, probablemente más sólida.

La segunda es que el país cuenta con un gran mercado interno. Según las encuestas internas realizadas junto a las ETs, éstas tienen por estrategia preservar las cuantiosas inversiones realizadas durante el proceso sustitutivo, y mantener o ampliar su participación en ese mercado, cuyas dimensiones potenciales la hace igualmente atractiva (CEPAL, 1993a).

Se debe mencionar también el hecho de que en ese momento de importantes decisiones estratégicas de ajuste por parte de las ETs en Brasil, el país disfrutaba de un nuevo e importante impulso dado por la expansión del comercio en el marco del Mercosur. La integración viabilizó la ampliación de las escalas de producción y reforzó en las ETs la decisión de mantener una base productiva sólida en el eje São Paulo-Buenos Aires.

Por áltimo, cabe una mención a los determinantes institucionales. Contrastando con los años ochenta, en que se mantuvo el marco regulatorio básico del período de sustitución de importaciones, los cambios institucionales han sido muy fuertes en los últimos años. Entre ellos, destacan la apertura comercial, la eliminación de la mayoría de los incentivos y subsidios a la industria y a las exportaciones y la privatización de las empresas en el sector manufacturero. No cabe duda que esos cambios han sido un importante elemento determinante del ajuste de las empresas desde 1990, además de la crisis.

Sin embargo, dado que la apertura de la economía es un proceso muy reciente, el caso de la reestructuración manufacturera en Brasil debe analizarse con cautela. Como es sabido, los resultados de la liberalización comercial suelen tomar tiempo para madurar, sobre todo en una economía compleja como la brasileña. No es imposible que el grado de desindustrialización en los próximos años vaya más allá de lo que se recomendaría a partir de consideraciones sobre la capacidad que tiene la economía de lograr dinámicamente la competitividad. Esa inquietud se reforzaría en la medida en que prosiga la crisis macroeconómica, y que se aprecie el tipo de cambio como parte de los esfuerzos antiinflacionarios. ${ }^{16}$

${ }^{16}$ De acuerdo con lo observado en la nota 13 en la introducción a esta subsección sobre Brasil, el análisis aquí presentado es anterior a la apreciación cambiaria que siguió al Plan Cardoso. Dependiendo de la evolución del tipo de cambio, este análisis podrá verse como muy optimista en el futuro. 


\section{V}

\section{Conclusión}

En este artículo se enfoca la reestructuración industrial, en Chile a partir de 1973, en Argentina desde 1978, en México desde 1982 y en Brasil desde 1981, fechas que corresponden al año siguiente al término del "modelo" de crecimiento por sustitución de importaciones.

El estudio comparado de las cuatro experiencias se funda en tres argumentos: primero, que en los cuatro casos se siguieron sendas o estilos muy distintos; segundo, que esa diferenciación obedeció en buena medida a la forma en que las ETs actuaron en cada país, o sea, a su reacción ante las situaciones de crisis y de apertura externa; y, tercero, que tanto la reestructuración industrial como las reacciones de las ETs fueron determinadas por la confluencia de tres conjuntos de factores: estructurales, macroeconómicos e institucionales, los cuales son específicos de cada país.

Por razones de espacio, no haremos aquí una síntesis de lo analizado en este artículo respecto de las transformaciones que experimentaron los cuatro países en la composición del producto, la inserción internacional, y la productividad del trabajo.

En consecuencia, pasaremos de inmediato a referirnos al aspecto más preocupante y polémico, que es la actuación del capital extranjero en la reestructuración industrial latinoamericana a partir de la crisis y de la apertura comercial, vale decir, cómo se vieron afectados los complejos metalmecánico y electroelectrónico. Aunque con marcadas diferencias entre los países, y si se excluyen la industria automotriz y una que otra rama de menor importancia, en general la evolución de este sector representó una inflexión de la tendencia histórica a aproximarse a los perfiles industriales de los países desarrollados.

Cabe aquí retomar el esquema analítico utilizado en el artículo para explicar las diferencias de comportamiento de las ETs según los países, de modo de resumir lo que sucedió con esas ramas. Con dicho esquema (gráfico 1) se tuvo el propósito de ir un poco más allá de la explicación genérica y tautológica de la falta de competitividad que afectará a la larga a las ETs ante la apertura comercial, resultante del modelo de sustitución de importaciones, de insuficiencias de escala y de recursos sistémicos; y se matizó el análisis mediante el ordenamiento de los ele- mentos estructurales, macroeconómicos e institucionales que dieron lugar a los procesos de los distintos países en sus especificidades.

En el caso de los sectores metalmecánico y electroelectrónico mencionados, es evidente que ante la alternativa que ofrecía la apertura a las empresas transnacionales de contraer sus actividades o invertir en modernización para competir a nivel internacional, éstas optaron frecuentemente por lo primero. Sin embargo, como las diferencias de comportamiento en los cuatro países fueron muy importantes, es posible caracterizar cuatro sendas o estilos muy distintos de reestructuración industrial.

El estilo de reestructuración industrial de Chile se ha definido en este artículo como de "desindustrialización con reorientación hacia afuera". Su estructura productiva relativamente simple, unida a la apertura rápida y acompañada de un tipo de cambio bajo provocaron una fuerte contracción del complejo metalmecánico/electroeletrónico en los años setenta, encabezada por varias ETs que desistieron de seguir produciendo desde el país. El tamaño del mercado interno era insuficiente para que la industria metalmecánica recuperara el peso que tenía hasta principios de los años setenta. Sin embargo, las condiciones macroeconómicas favorables y el fuerte crecimiento sostenido por una década estaban sentando las bases para una cierta recuperación, en ramas de menor sofisticación tecnológica y dedicadas al mercado doméstico.

$\mathrm{Al}$ estilo argentino de reestructuración lo llamamos de "desofisticación importadora", expresión utilizada en el artículo para reflejar a la vez la contracción de las ramas metalmecánica y electroelectrónica y la fuerte expansión de las importaciones de esos bienes industriales. También en ese país hubo una fuga de varias ETs, y una reducción de otras, principalmente en la rama metalmecánica. Al final del proceso de sustitución existía una industria metalmecánica de tamaño mediano con algún grado de diversificación. La profundidad de la crisis macroeconómica de los años ochenta dio continuidad al proceso de desindustrialización iniciado con la crisis y la política económica de la segunda mitad de los años setenta, en que se conjugaron recesión, apertura comercial y apreciación cambiaria. En la fase actual de 
reactivación economica la recuperación ha sido muy fuerte en los sectores electrodomésticos y automotriz, en este último con el importante aliento del acuerdo sectorial suscrito en el ámbito del Mercosur. Varias de las ETs que se habían retirado del país están volviendo. Sin embargo, en general las ETs pasaron a producir con coeficientes de importación mucho mayores, y en los sectores de los electrodomésticos y de la electrónica de consumo, la producción está prácticamente reducida al ensamblaje, en Tierra del Fuego.

La senda de reestructuración seguida por México se distingue fuertemente de las de los otros casos examinados en este artículo, y la define su integración con la economía de los Estados Unidos, bajo el claro liderazgo de las ETs. Una estructura productiva $y$ un mercado interno de proporciones razonables, las inversiones destinadas al mercado norteamericano y la mantención de un tipo de cambio alto en el período inicial de la apertura comercial compensaron en los años ochenta, en el caso de importantes ramas, la influencia muy negativa de la severa crisis macroeconómica. Entre dichas ramas destacan la automotriz y la "maquila". Las ramas más afectadas incluyeron, entre otras, varios segmentos de la industria de bienes de capital, con lo que se produjo una inflexión de una tendencia anterior a formar un subsector de maquinarias pesadas en ese país. Asimismo, la acelerada liberalización comercial y la fuerte apreciación cambiaria a partir de 1988 contribuyeron a que en muchos segmentos de la industria de bienes de capital y de consumo no duraderos, el debilitamiento provocado por la crisis de los años ochenta se confirmara durante la reciente fase de recuperación económica.

El estilo de reestructuración industrial de Brasil ha sido denominado "defensivo", dada la tendencia a preservar una parcela mayor de la estructura productiva heredada del período de sustitución de importaciones. En buena medida, esa tendencia se funda en la estrategia de las ETs de preservar sus cuantiosas inversiones anteriores y su fuerte participación en el mercado doméstico. La relativa complejidad de las industrias metalmecánica y electroelectrónica al final de la industrialización sustitutiva, el gran tamaño del mercado interno y el cierre de la economía, permitieron a la industria pesada sobrevivir en los años ochenta, en condiciones de estancamiento y de pasividad tecnológica. Por otro lado, la relativa solidez estructural y el tipo de cambio relativamente alto y estable le permitió evitar (hasta el primer semestre de 1994) una fuerte desindustrialización en las difíciles condiciones de apertura y crisis macroeconómica.
Actualmente está en proceso una fuerte recuperación de la industria automotriz, acompañada de importantes inversiones. Sin embargo, la reciente apertura redundó en la contracción de algunas ramas de gran intensidad tecnológica, como las de computadores, componentes electrónicos y de algunos bienes de capital seriados. La crisis y las bajas tasas de inversión dificultan hasta el momento estimar el grado de competitividad relativa actual de los sectores de bienes de capital.

En resumen, el análisis de la reestructuración industrial en los cuatro países estudiados, revela un deterioro del grado de complejidad en los sistemas industriales, lo que los aleja de la estructura de los países de la OCDE. Este proceso no se dio con la misma intensidad en todos los países, destacándose Brasil como el de mayor capacidad de conservación de su estructura anterior, que ya era más sofisticada. El caso de México es especial porque el deterioro se produjo simultáneamente con el fortalecimiento de algunos sectores de gran dinamismo comercial y tecnologico, en particular la industria automotriz. Argentina y Chile tuvieron un desempeño claramente inferior a Brasil y México en lo que se refiere a la evolución de la composición de la producción y de las exportaciones del sector manufacturero.

La actuación de las empresas transnacionales fue decisiva en la evolución de las cuatro estructuras productivas - como vimos, a través de "estilos" de comportamiento muy diferentes. Se puede concluir que dicha actuación fue más favorable $\longrightarrow$ o menos desfavorable - para las perspectivas futuras de desarrollo industrial de México y Brasil que de Chile y Argentina.

De lo expuesto anteriormente se llega a una conclusión básica. El alejamiento respecto de los perfiles industriales más desarrollados, junto a la relativa desindustrialización de los complejos metalmecánico y electroelectrónico puede tener una implicancia negativa para el futuro del desarrollo económico de los países latinoamericanos. Dichos sectores son los que poseen, en todas partes, el mayor poder de generación de crecimiento, y el mayor dinamismo tecnológico y poder de difusión de progreso técnico al resto de la economía. También son los sectores en los cuales la demanda se amplía rápidamente, sobre todo en los países en desarrollo, lo que puede tener consecuencias muy graves para el movimiento del balance de pagos.

Para finalizar, y en relación con lo anterior, cabe hacer un comentario sobre las "políticas para atraer capital extranjero": la capacidad de atraer capital extranjero hacia el sector manufacturero depende de que 
existan condiciones favorables para las actividades de su preferencia, las que en general son aquellas que utilizan intensivamente la tecnología y el comercio internacional. Si un gobierno latinoamericano rechaza la idea de que el sistema industrial siga incorporando tales actividades, y les da tratamientos de "choque"
- por ejemplo, a través de una liberalización comercial rápida asociada a una apreciación cambiaria- está optando implícitamente por rechazar la producción local por parte de las empresas transnacionales, reducir el impacto de su papel de incorporadoras y difusoras de tecnologías, y de promotoras de exportaciones.

\section{Bibliografía}

Agacino, R, G. Rivas y E. Román (1992): Apertura y eficiencia productiva: la experiencia chilena 1975-1989, Documentos de trabajo, $\mathrm{N}^{\circ} 113$, Washington, D.C., Banco Interamericano de Desarrollo (BID).

Aguilera M. y J. Becar (1991): Antecedentes y evolución del sector industrial manufacturero en Chile 1980-1989, Santiago de Chile, Universidad de Chile, Departamento de Economía, Facultad de Ciencias Económicas y Administrativas.

Aspiazu, D. (en prensa): Las empresas transnacionales en una economía en transición. La experiencia argentina en los años ochenta, Estudios e Informes de la CEPAL, $\mathbf{N}^{\circ}$ 91, Santiago de Chile, Comisión Económica para América Latina y el Caribe (CEPAL), mimeo.

Barros O. (1993): Oportunidades abertas para o Brasil face aos fluxos globais de investimento de risco e de capitais financeiros nos anos 90, Campinas, Brasil, Universidade Estadual de Campinas (UNICAMP)/Universidad Federal de Rio de Janeiro, mimeo.

Beccaria, L.A. y B. Kosacoff (cons.) (1994): La industria argentina: de la substitución de importaciones a la convertibilidad (LC/R. 1454), Santiago de Chile, CEPAL.

Calderón A. (1994a): FDI in Latin America and the Caribbean: Policy elements and results, Foreign Direct Investment in Developing Countries: The Case of Latin America, Madrid, Instituto de Relaciones Europeo-Latinoamericanas (IRELA).

(1994b): El papel de las empresas transnacionales en la transformación productiva de la industria manufacturera en Chile, Santiago de Chile, CEPAL, mimeo.

Casar, J. (1993): La competitividad de la industria manufacturera mexicana 1980-1990, El trimestre económico, vol. 60 (1), N 237 , México, D.F., Fondo de Cultura Económica.

(1994): Un balance de la transformación industrial en México, Santiago de Chile, CEPAL, mimeo.

Casar, J. y otros (1989): La organización industrial en México, México, D.F., Siglo XXI.

Castillo, M., M. Dini y C. Maggi (1994): Reorganización industrial y estrategias competitivas en Chile, Santiago de Chile, CEPAL, mimeo.

CEPAL (1991): Informe sobre la modernización industrial en México (LC/L.613), Santiago de Chile.

(1993a): Transnational Corporations and the Manufacturing Sector in Brazil (LC/R. 1261), Santiago de Chile, Unidad Conjunta CEPAL/DDES (Departamento de Desarrollo Económico y Social) sobre Empresas Transnacionales.

(1993b): Adjusting for survival: Foreign and national manufacturing firms in Brazil in the early 1990s, Santiago de Chile, Unidad Conjunta CEPAL/DDES (Departamento de Desarrollo Económico y Social) sobre Empresas Transnacionales, mimeo.

(1993c): Organización industrial, competitividad internacional y política pública en la década de los años noventa (LC/R. 1329), Santiago de Chile.

CET (Centro de las Naciones Unidas sobre las Empresas Transnacionales) (1992): Foreign Direct Investment and Industrial
Restructuring in México, UNCTC Current Studies, series A, $\mathrm{N}^{\circ} 18$, Nueva York. Publicación de las Naciones Unidas, $N^{\circ}$ de venta E.92.II.A.9.

Chudnovski, D., A. López y F. Porta (1993): Ajuste estructural y estrategias empresariales en Argentina, Brasil y México. Un análisis comparativo de la industria petroquímica y de máquinas herramientas, Documentos de trabajo, $\mathrm{N}^{\circ} 11$, Buenos Aires, Centro de Investigaciones para la Transformación (CENIT).

(1994): La nueva inversión estranjera directa en la Argentina. Privatizaciones, mercado interno e integración regional, Documentos de trabajo, $\mathrm{N}^{\circ} 15$, Buenos Aires, CENIT.

Coutinho, L. y J.C. Ferraz (1994): Estudo da competividade da industria brasileira, Campinas, Brasil, UNICAMP/PAPIRUS.

Díaz, A. (1994): Dinámicas del cambio tecnologico en la industria chilena, Santiago de Chile, mimeo.

Dunning, J.H. (1973): The determinants of international production, Oxford Economic Papers, vol. 25, $\mathrm{N}^{\circ} 3$, Oxford, Gran Bretaña, Oxford University Press.

Dussel Peters, E. (1993): Cambio estructural y potencialidades de crecimiento del sector manufacturero en México (1982-1991), México, D.F., mimeo.

Erber, F. y R. Vermulm (1992): Ajuste estrutural e estratégias empresariais - um estudo de caso dos setores petroquimico e de máquinas-ferramenta no Brasil, Rio de Janeiro, Instituto de Pesquisa Aplicada (IPEA), mimeo.

Fritsch, W. y G. Franco (1991): Foreign Direct Investment in Brazil: Its Impact on Industrial Restructuring, París, Organización de Cooperación y Desarrollo Económicos (OCDE).

Hymer, S. (1976): The International Operations of National Firms: A Study of Direct Investment, Cambridge, Mass., MIT Press, [la primera edición fue publicada en 1960].

IEI (Instituto de Estudios Internacionales de la Universidad Federal de Rio de Janeiro)/UNICAMP (1993): Estudo da competitividade da indústria brasileira, relatório final, Brasilia, Brasil, mimeo.

Katz, J. (1974): Importación de tecnología, aprendizaje e industrialización dependiente, México, D.F., Fondo de Cultura Económica.

(1994): Cambios estructurales en la industria manufacturera argentina, estudios comparativos entre la etapa sustitutiva y el escenario industrial contemporáneo, Santiago de Chile, CEPAL, mimeo.

Kosacoff, B. (org.) (1993): El desafio de la competitividad. La industria argentina en transformación, Buenos Aires, Alianza Editorial, S.A.

Kosacoff, B. y G. Bezchinsky (1993): De la sustitución de importaciones a la globalización. Las empresas transnacionales en la industria argentina, B. Kosacoff (org.), El desafío de la competitividad. La industria argentina en transformación, Buenos Aires, Alianza Editorial, S.A.

Peres, W. (1990): Foreign Direct Investment and Industrial Development in México, París, OCDE.

Ros, J. (1991): Industrial Organization and Comparative Advantage in Mexico's Manufacturing Trade, Working paper, 
$\mathrm{N}^{\circ} 155$, Notre Dame, IN, Estados Unidos, The Hellen Kellog Institute for International Studies, University of Notre Dame.

Rozas, P. (cons.) (1992): Inversión extranjera y empresas transnacionales en la economía de Chile (1974-1989), Estudios e informes de la CEPAL, $N^{\circ} 85$ (LC/G.1677-P), Santiago de Chile, CEPAL. Publicación de las Naciones Unidas, $\mathrm{N}^{\mathbf{0}}$ de venta S.92.II.G.7.
Suzigan, W. (1991): Situaçã̃o atual da indústria brasileira e implicações para a política industrial, Planejamento e políticas públicas, $\mathrm{N}^{\circ}$ 6, Brasilia, Brasil, Instituto de Pesquisa Económica Aplicada (IPEA).

Unger, K. , L.C. Saldaña, J. Jasso y G. Durand (1992): Ajuste estructural y estrategias empresariales en las industrias petroquímicas y de máquinas herramientas en México, México, D.F., mimeo. 